\title{
CHERNOFF APPROXIMATION FOR SEMIGROUPS GENERATED BY KILLED FELLER PROCESSES AND FEYNMAN FORMULAE FOR TIME-FRACTIONAL FOKKER-PLANCK-KOLMOGOROV EQUATIONS
}

\author{
YANA A. BUTKO
}

\begin{abstract}
Semigroups, generated by Feller processes killed upon leaving a given domain, are considered. These semigroups correspond to Cauchy-Dirichlet type initial-exterior value problems in this domain for a class of evolution equations with (possibly non-local) operators. The considered semigroups are approximated by means of the Chernoff theorem. For a class of killed Feller processes, the constructed Chernoff approximation converts into a Feynman formula, i.e. into a limit of $n$-fold iterated integrals of certain functions as $n \rightarrow \infty$. Representations of solutions of evolution equations by Feynman formulae can be used for direct calculations and simulation of underlying stochasstic processes. Further, a method to approximate solutions of time-fractional (including distributed order time-fractional) evolution equations is suggested. This method is based on connections between time-fractional and time-nonfractional evolution equations as well as on Chernoff approximations for the latter ones. Moreover, this method leads to Feynman formulae for solutions of time-fractional evolution equations. To illustrate the method, a class of distributed order time-fractional diffusion equations is considered; Feynman formulae for solutions of the corresponding Cauchy and Cauchy-Dirichlet problems are obtained.
\end{abstract}

Keywords: Chernoff approximation, Feynman formula, approximation of operator semigroups, approximation of transition probabilities, killed Feller processes, initial-exterior value problems, fractional diffusion, distributed order time-fractional equations, time-fractional Fokker-Planck-Kolmogorov equations.

\section{Contents}

1. Introduction

2. Notations and preliminaries

2.1. Chernoff theorem and Feynman formulae

2.2. Killed Feller processes and their generators

2.3. Distributed-order fractional derivatives

3. Chernoff approximation of semigroups generated by some killed Feller processes

3.1. General approach

3.2. Feynman Formula for semigroups generated by a class of killed Feller processes

4. Approximation of solutions and Feynman formulae for time-fractional Fokker-Planck-Kolmogorov equations

4.1. Approximation of solutions for distributed order time-fractional Fokker-Planck-Kolmogorov equations

4.2. Feynman formula solving the Cauchy problem for a class of time-fractional diffusion equations 
4.3. Feynman formula solving the Cauchy-Dirichlet problem for a class of time-fractional diffusion equations

Acknowledgements

References

\section{INTRODUCTION}

Classical results of Analysis and Stochastics provide deep connections between such mathematical objects as operator semigroups, evolution equations, path integrals and Markov processes. A strongly continuous operator semigroup $\left(T_{t}\right)_{t \geq 0}$ with a given generator $L$ on a given Banach space $X$ allows to solve an initial (or initial-boundary) value problem for the corresponding evolution equation $\frac{\partial f}{\partial t}=L f$ with the initial value $f_{0}$ by the identity $f(t)=T_{t} f_{0}$. On the other hand, if $\left(\xi_{t}\right)_{t \geq 0}$ is a Markov process with generator $L$, the semigroup $\left(T_{t}\right)_{t \geq 0}$ defines transition probability $P(t, x, d y)$ of this process by the formula $T_{t} f_{0}(x)=\int f_{0}(y) P(t, x, d y)=$ $\mathbb{E}^{x}\left[f_{0}\left(\xi_{t}\right)\right]$. The last expression gives a stochastic representation for the solution of the above mentioned evolution equation in $X$. A basic example is a $d$-dimensional Brownian motion whose transitional probability $P(t, x, d y)$ is given by the Gaussian exponent $P(t, x, d y):=(2 \pi t)^{-d / 2} \exp \left(-\frac{|x-y|^{2}}{2 t}\right) d y$, and the corresponding evolution equation is the heat equation $\frac{\partial f}{\partial t}=\frac{1}{2} \Delta f$ with the Laplace-operator $\Delta$.

Usually, however, the semigroup $\left(T_{t}\right)_{t \geq 0}$ or, respectively, the transition probability $P(t, x, d y)$ is not known explicitly. One of analytical approaches to deal with unknown semigroups / transitional probabilities is the use of the Chernoff Theorem (which is a very broad generalization of the classical Daletskii-Lie-Trotter formula). This theorem provides conditions for a family (just a family, not a semigroup!) of bounded linear operators $(F(t))_{t \geq 0}$ to approximate the semigroup $\left(T_{t}\right)_{t \geq 0}$ with a given generator $L$ via the formula $T_{t}=\lim _{n \rightarrow \infty}[F(t / n)]^{n}$. This formula is called Chernoff approximation of the semigroup $\left(T_{t}\right)_{t \geq 0}$ by the family $(F(t))_{t \geq 0}$. The method of Chernoff approximation has the following advantage: if the family $(F(t))_{t \geq 0}$ is given explicitly, the expressions $[F(t / n)]^{n}$ can be directly used for calculations and hence for approximation of solutions of corresponding evolution equations, for computer modelling of considered dynamics, for approximation of transition probabilities of underlying Markov processes and hence for simulation of these processes. Moreover, if all operators $F(t)$ are integral operators with elementary kernels (or pseudo-differential operators with elementary symbols), the identity $T_{t}=\lim _{n \rightarrow \infty}[F(t / n)]^{n}$ leads to representation of the semigroup $\left(T_{t}\right)_{t \geq 0}$ by limits of $n$-fold iterated integrals of elementary functions when $n$ tends to infinity. Such representations are called Feynman formulae. Feynman formulae have an additional advantage. Namely, the limits in Feynman formulae usually coincide with functional (or path) integrals with respect to probability measures (i.e., with Feynman-Kac formulae providing stochastic representations for solutions of the corresponding evolution equations) or with respect to Feynman pseudomeasures (i.e., with Feynman path integrals). Therefore, representations of evolution semigroups by Feynman formulae allow to establish new Feynman-Kac formulae and give an additional tool to calculate path integrals numerically. Note that different Feynman formulae for the same semigroup allow to establish relations between different path integrals. This leads, in particular, to some "change-of-variables" rules, connecting certain Feynman-Kac formulae and Feynman path integrals (see the papers [19, 7]). Chernoff approximation can be understood in some particular cases also 
as a construction of Markov chains approximating a given Markov process [9] and as a numerical path integration method for solving corresponding PDEs/SDEs [23].

It is worth to mention that the method of Chernoff approximation has a wide range of applications. For example, this method has been used to investigate Schrödinger type evolution equations in [71, 66, 74, 41, 30, 84, 81, 83, stochastic Schrödinger type equations have been studied in [58, 57, 59, 34. Second order parabolic equations related to diffusions in different geometrical structures (e.g., in Eucliean spaces and their subdomains, Riemannian manifolds and their subdomains, metric graphs, Hilbert spaces) have been studied, e.g., in [19, 15, 69, 14, 67, 82, 170, 7, [20, 90, 18, 89, 17, 13, 12, 86, 11, 10, 85, 56. Evolution equations with non-local operators generating some Markov processes in $\mathbb{R}^{d}$ have been considered in 16, 19, 21, 22. Evolution equations with the Vladimirov operator (this operator is a $p$-adic analogue of the Laplace operator) have been investigated in [79, 80, 78, 77, 76. Feynman formulae as a method of averaging of random Hamiltonians have been discussed in [61, 60]. In the present note, the method of Chernoff approximation is applied to investigation of initial-exterior value problems for evolution equations with non-local operators in a given subdomain $G$ of $\mathbb{R}^{d}$. Such problems correspond to governing equations for Feller processes, killed upon leaving the domain $G$. For a special class of killed Feller processes, solutions of the corresponding initial-exterior problems are represented by Feynman formulae.

Fractional derivatives are natural extensions of their integer-order analogues (see, e.g., 53, 75]). Evolution equations with partial derivatives of fractional order (fractional evolution equations), in particular, time- (and, possibly, space-) fractional diffusion equations (modelling anomalous diffusion) have been applied to problems in physics, chemistry, biology, medicine, finance, hydrology and other areas (see, e.g., [48, 50, 51, 53, 75] and references therein). Many time-fractional evolution equations serve as governing equations for stochastic processes. However, the processes, whose marginal density function evolves in time according to a given time-fractional evolution equation, are usually non-Markovian (and hence, there is no semigroup structure behind the equation) and are non-uniquely defined by this marginal density function (therefore, very different stochastic representations for a solution of a given fractional evolution equation are possible, see, e.g., [1, 37, 55]). The absence of the semigroup property for solutions of time-fractional evolution equations does not allow to apply the method of Chernoff approximation for such equations directly. Nevertheless, several relations exist between time-fractional and "standard" (time-non-fractional) evolution equations: via a kind of subordination (see, e.g., 68, 73, 50, 3, 55]) and via higher order operators (see, e.g., 62, 63, 4, 64, 31]). These relations allow to construct some approximations for solutions of such timefractional evolution equations via Chernoff approximations for solutions of some related "standard" evolution equations. In this note, we present approximations for solutions of (a class of) time-fractional evolution equations using their connection to time-non-fractional equations via a kind of subordination. In particular, Feynman formulae are obtained for solutions of Cauchy and Cauchy-Dirichlet problems for distributed order time-fractional diffusion equations.

\section{Notations AND PRELIMINARIES}

2.1. Chernoff theorem and Feynman formulae. In the sequel, the following version of the Chernoff theorem is used (cf. [26, 27]).

Theorem 2.1. Let $(F(t))_{t \geq 0}$ be a family of bounded linear operators on a Banach space $X$. Assume that

(i) $F(0)=\mathrm{Id}$,

(ii) $\|F(t)\| \leq e^{w t}$ for some $w \in \mathbb{R}$ and all $t \geq 0$, 
(iii) the limit $L \varphi:=\lim _{t \rightarrow 0} \frac{F(t) \varphi-\varphi}{t}$ exists for all $\varphi \in D$, where $D$ is a dense subspace in $X$ such that $(L, D)$ is closable and the closure $(L, \operatorname{Dom}(L))$ of $(L, D)$ generates a strongly continuous semigroup $\left(T_{t}\right)_{t \geq 0}$.

Then the semigroup $\left(T_{t}\right)_{t \geq 0}$ is given by

$$
T_{t} \varphi=\lim _{n \rightarrow \infty}[F(t / n)]^{n} \varphi
$$

for all $\varphi \in X$, and the convergence is locally uniform with respect to $t \geq 0$.

The formula (1) is called Chernoff approximation of the semigroup $\left(T_{t}\right)_{t \geq 0}$ by the family $(F(t))_{t \geq 0}$. Any family $(F(t))_{t \geq 0}$, satisfying the assumptions (i)-(iii) of the Chernoff theorem 2.1 with respect to a given semigroup $\left(T_{t}\right)_{t \geq 0}$, is called Chernoff equivalent to this semigroup.

Definition 2.1. A Feynman formula is a representation of a solution of an initial (or initial-boundary) value problem for an evolution equation (or, equivalently, a representation of the semigroup solving the problem) by a limit of $n$-fold iterated integrals of some functions as $n \rightarrow \infty$.

One should not confuse the notions of Chernoff approximation and Feynman formula. On the one hand, not all Chernoff approximations can be directly interpreted as Feynman formulae since, generally, the operators $(F(t))_{t \geq 0}$ do not have to be neither integral operators, nor pseudo-differential operators. On the other hand, representations of solutions of evolution equations in the form of Feynman formulae can be obtained by different methods, not necessarily via the Chernoff Theorem. And such Feynman formulae may have no relations to any Chernoff approximation, or their relations may be quite indirect (see, e.g., Feynman formulae (16), (27), (31) below).

2.2. Killed Feller processes and their generators. In this Subsection, we follow the exposition of 8 and 2 . Let $Q$ be a locally compact separable metric space. Let $C_{0}(Q)$ be the set of all bounded continuous functions $\varphi$ on $Q$ such that $\forall \varepsilon>0 \exists$ a compact $K_{\varphi}^{\varepsilon} \subset Q$ with $|\varphi(q)|<\varepsilon \forall q \notin K_{\varphi}^{\varepsilon}$. The set $X:=C_{0}(Q)$ is a Banach space endowed with the supremum-norm. A semigroup of bounded linear operators $\left(T_{t}\right)_{t \geq 0}$ on the Banach space $X$ is called Feller semigroup if it is a strongly continuous semigroup, it is positivity preserving (i.e. $T_{t} \varphi \geq 0$ for all $\varphi \in X$ with $\varphi \geq 0$ ) and it is sub-Markovian (i.e. $T_{t} \varphi \leq 1$ for all $\varphi \in X$ with $\varphi \leq 1$ ). Its generator (called Feller generator $)$ is defined by $\operatorname{Dom}(L):=\left\{\varphi \in X \mid \lim _{t \rightarrow 0} \frac{T_{t} \varphi-\varphi}{t}\right.$ exists in $\left.X\right\}$, $L \varphi:=\lim _{t \rightarrow 0} \frac{T_{t} \varphi-\varphi}{t} \quad \forall \varphi \in \operatorname{Dom}(L)$. Let $(\Omega, \mathcal{F}, \mathbb{P})$ be a probability space with a filtration $\left(\mathcal{F}_{t}\right)_{t \geq 0}$, and let $\left(\xi_{t}\right)_{t \geq 0}$ be a temporally homogeneous Markov process with state space $(Q, \mathcal{B}(Q))$, where $\mathcal{B}(Q)$ is the Borel sigma-algebra of $Q$. The process $\left(\xi_{t}\right)_{t \geq 0}$ is called Feller process if its transition semigroup $\left(T_{t}\right)_{t \geq 0}$, $T_{t} \varphi(x):=\int_{Q} \varphi(y) \mathbb{P}^{x}\left(\xi_{t} \in d y\right) \equiv \mathbb{E}^{x}\left[\varphi\left(\xi_{t}\right)\right]$, is a Feller semigroup. Using a version of the Riesz representation theorem, one can extend a Feller semigroup $\left(T_{t}\right)_{t \geq 0}$ to a sub-Markovian semigroup on the space $B_{b}(Q)$ of bounded Borel functions. A sub-Markovian semigroup $\left(T_{t}\right)_{t \geq 0}$ (on $B_{b}(Q)$ ) is called strong Feller semigroup if $T_{t}: B_{b}(Q) \rightarrow C_{b}(Q)$ for all $t>0$. Note that a strong Feller semigroup need not be Feller and vice versa. Some conditions on Feller semigroups to be strong Feller can be found in Lemma 1.12, Theorem 1.14 and Theorem 1.15 of [8]. The process $\left(\xi_{t}\right)_{t \geq 0}$ (resp., the semigroup $\left(T_{t}\right)_{t \geq 0}$ with $T_{t} \varphi(x):=\mathbb{E}^{x}\left[\varphi\left(\xi_{t}\right)\right]$ ) is called doubly Feller if it is both Feller and strong Feller.

Let $Q=\mathbb{R}^{d}$ and $X=C_{0}\left(\mathbb{R}^{d}\right)$. Let $\left(\xi_{t}\right)_{t \geq 0}$ be a Feller process on $\mathbb{R}^{d},\left(T_{t}\right)_{t \geq 0}$ be the corresponding Feller semigroup and $(L, \operatorname{Dom}(L))$ be its Feller generator. We 
define the pointwise generator $\left(L_{p}, \operatorname{Dom}\left(L_{p}\right)\right)$ of $\left(T_{t}\right)_{t \geq 0}$ by

$$
\begin{aligned}
& \operatorname{Dom}\left(L_{p}\right):=\left\{\varphi \in X \mid \exists g \in X: \lim _{t \rightarrow 0} \frac{T_{t} \varphi(x)-\varphi(x)}{t}=g(x) \forall x \in \mathbb{R}^{d}\right\}, \\
& L_{p} \varphi(x):=\lim _{t \rightarrow 0} \frac{T_{t} \varphi(x)-\varphi(x)}{t}=g(x) \quad \forall \varphi \in \operatorname{Dom}\left(L_{p}\right), x \in \mathbb{R}^{d} .
\end{aligned}
$$

Then $(L, \operatorname{Dom}(L))=\left(L_{p}, \operatorname{Dom}\left(L_{p}\right)\right)$ by Theorem 1.33 of $[8]$. Moreover, i $C_{c}^{\infty}\left(\mathbb{R}^{d}\right) \subset$ $\operatorname{Dom}(L)$, then we have also $C_{0}^{2}\left(\mathbb{R}^{d}\right):=\left\{\varphi \in C^{2}\left(\mathbb{R}^{d}\right): \partial^{\alpha} \varphi \in C_{0}\left(\mathbb{R}^{d}\right),|\alpha| \leq 2\right\} \subset$ $\operatorname{Dom}(L)$. And $L \varphi(x)$ is given for each $\varphi \in C_{0}^{2}\left(\mathbb{R}^{d}\right)$ and each $x \in \mathbb{R}^{d}$ by the folowing formula:

$$
\begin{aligned}
L \varphi(x)= & -C(x) \varphi(x)-B(x) \cdot \nabla \varphi(x)+\operatorname{tr}(A(x) \operatorname{Hess} \varphi(x)) \\
& +\int_{y \neq 0}\left(\varphi(x+y)-\varphi(x)-\frac{y \cdot \nabla \varphi(x)}{1+|y|^{2}}\right) N(x, d y),
\end{aligned}
$$

where Hess $\varphi$ is the Hessian matrix of second order partial derivatives of $\varphi$; as well as $C(x) \geq 0, B(x) \in \mathbb{R}^{d}, A(x) \in \mathbb{R}^{d \times d}$ is a symmetric positive semidefinite matrix and $N(x, \cdot)$ is a Radon measure on $\mathbb{R}^{d} \backslash\{0\}$ with $\int_{y \neq 0}|y|^{2}\left(1+|y|^{2}\right)^{-1} N(x, d y)<\infty$ for each $x \in \mathbb{R}^{d}$. Therefore, $L$ is an integro-differential operator on $C_{0}^{2}\left(\mathbb{R}^{d}\right)$. And this operator is non-local if $N \neq 0$. This class of generators $L$ includes, in particular, fractional Laplacians $L=-(-\Delta)^{\alpha / 2}$ and relativistic Hamiltonians $\sqrt[\alpha]{(-\Delta)^{\alpha / 2}+m(x)}$, $\alpha \in(0,2), m>0$.

Let now $G \subset \mathbb{R}^{d}$ be a bounded domain (connected open set) and let $Y:=C_{0}(G)$ be the set of all continuous functions on $G$ that tend to zero as $x \in G$ approaches the boundary $\partial G$. Then $Y$ is a Banach space with the supremum norm $\|\cdot\|_{Y}$, $\|\varphi\|_{Y}:=\sup _{x \in G}|\varphi(x)|$. For a Feller process $\left(\xi_{t}\right)_{t \geq 0}$ on $\mathbb{R}^{d}$, we define the first exit time from $G$ by

$$
\tau_{G}:=\inf \left\{t>0: \xi_{t} \notin G\right\} .
$$

Let $\left(\xi_{t}^{o}\right)_{t \geq 0}$ denote the killed process on $G$, i.e.,

$$
\xi_{t}^{o}= \begin{cases}\xi_{t}, & t<\tau_{G}, \\ \partial, & t \geq \tau_{G},\end{cases}
$$

where $\partial$ denotes a cemetery point (i.e. an isolated point $\partial \notin G$ added to the state space $G$ ). We say that a boundary point $x \in \partial G$ is regular for $G$ if $\mathbb{P}^{x}\left(\tau_{G}=0\right)=1$. We say that $G$ is regular if every point $x \in \partial G$ is regular Let $\left(\xi_{t}\right)_{t \geq 0}$ be a doubly Feller process on $\mathbb{R}^{d}$ and $G$ be regular. Then $\left(T_{t}^{o}\right)_{t \geq 0}$, such that

$$
T_{t}^{o} \varphi(x):=\mathbb{E}^{x}\left[\varphi\left(\xi_{t}^{o}\right)\right], \quad \varphi \in Y, x \in G,
$$

is a Feller semigroup on $Y$ (cf. Lemma 2.2 in [2]). Let $\left(L_{o}, \operatorname{Dom}\left(L_{o}\right)\right)$ be the Feller generator of $\left(T_{t}^{o}\right)_{t \geq 0}$ on $Y$. The operator $\left(L_{o}, \operatorname{Dom}\left(L_{o}\right)\right)$ is described in Proposition 2.1 below (cf. Thm. 2.3 and Lemma 2.6 in [2]). Note that each element $\varphi \in Y$ can be extended by zero outside $G$; this extension is again denoted by $\varphi$ and belongs to the space $X$.

Proposition 2.1. Let $\left(\xi_{t}\right)_{t \geq 0}$ be a doubly Feller process on $\mathbb{R}^{d},\left(T_{t}\right)_{t \geq 0}$ be the corresponding Feller semigroup and $(L, \operatorname{Dom}(L))$ be its Feller generator. Let $G \subset \mathbb{R}^{d}$ be a bounded regular domain. The generator of the killed Feller process $\left(\xi_{t}^{o}\right)_{t \geq 0}$ is characterized as follows

\footnotetext{
${ }^{1}$ The assumption $C_{c}^{\infty}\left(\mathbb{R}^{d}\right) \subset \operatorname{Dom}(L)$ is quite standard and holds in many cases, see, e.g., 8 .

${ }^{2}$ Due to Theorem 2.2. of 25, if a boundary point $x \in \partial G$ satisfies the external cone condition, then it is regular for the case when $\left(\xi_{t}\right)_{t \geq 0}$ is symmetric $\alpha$-stable. In particular, any Lipschitz domain is regular in this case.
} 
(i) $\operatorname{Dom}\left(L_{o}\right)=\left\{\varphi \in Y: L_{p} \varphi \in Y\right\}$, where $L_{p}$ is given by (2), $L_{p} \varphi \in Y$ means that $L_{p}$ is applied to the zero extension of $\varphi$ on $\mathbb{R}^{d}, L_{p} \varphi(x)$ exists for each $x \in G$ and the function $\left[x \mapsto L_{p} \varphi(x)\right]$ belongs to $Y$. Moreover, it holds that $L_{o} \varphi(x)=L_{p} \varphi(x)$ for all $\varphi \in \operatorname{Dom}\left(L_{o}\right)$ and the limit in (2) exists locally uniformly on $G$ (i.e., uniformly with respect to $x \in K$ for each compact $K \subset G)$.

(ii) Assume that $C_{c}^{\infty}\left(\mathbb{R}^{d}\right) \subset \operatorname{Dom}(L)$. Then, for each $\varphi \in C_{0}\left(\mathbb{R}^{d}\right) \cap C^{2}(G)$, we have

$$
L_{p} \varphi(x)=L \varphi(x), \quad \forall x \in G,
$$

where $L$ is the integro-differential operator given by the formula (3).

Remark 2.1. (i) The abstract Cauchy problem in $Y$ for the evolution equation $\frac{d f}{d t}=L_{o} f$ with an initial condition $f_{0} \in \operatorname{Dom}\left(L_{o}\right)$ can be interpreted as the following Cauchy-Dirichlet type initial-exterior value problem 3 :

$$
\begin{aligned}
& \frac{\partial f}{\partial t}(t, x)=L f(t, x), \quad t>0, x \in G, \\
& f(0, x)=f_{0}(x), \quad x \in G, \\
& f(t, x)=0, \quad t>0, \quad x \in \mathbb{R}^{d} \backslash G .
\end{aligned}
$$

And the function $f(t, x):=T_{t}^{o} f_{0}(x)$, extended by zero outside $G$, solves the problem for each $f_{0} \in \operatorname{Dom}\left(L_{o}\right)$ (e.g., by Theorem 4.1 .3 in [65]).

(ii) Let, additionally, the operator $(L, \operatorname{Dom}(L))$ in $X$ be a local operator outside $\bar{G}:=G \cup \partial G$, i.e. for each $x \in \mathbb{R}^{d} \backslash \bar{G}$ and each $\varphi_{1}, \varphi_{2} \in \operatorname{Dom}(L)$ such that $\varphi_{1}$ and $\varphi_{2}$ coincide on $\bar{G}$ and on some neighbourhood of $x$, one has $L \varphi_{1}(x)=L \varphi_{2}(x)$. For example, consider the integro-differential operator $L$ given by (3) with $N(x, d y)$ such that

$$
N(x, d y)= \begin{cases}1_{0<|y| \leq \operatorname{dist}(x, \partial G)}(y) N(x, d y), & x \in G, \\ 0, & x \in \mathbb{R}^{d} \backslash G .\end{cases}
$$

The integral part of such operator $L$ gives rise to the so-called censored processes in $G$, cf. 6, 72. If $L$ is local outside $\bar{G}$, the abstract Cauchy problem in $Y$ for the evolution equation $\frac{d f}{d t}=L_{o} f$ can be interpreted as the following Cauchy-Dirichlet problem:

$$
\begin{aligned}
& \frac{\partial f}{\partial t}(t, x)=L f(t, x), \quad t>0, x \in G, \\
& f(0, x)=f_{0}(x), \quad x \in G, \\
& f(t, x)=0, \quad t>0, \quad x \in \partial G .
\end{aligned}
$$

And again the function $f(t, x):=T_{t}^{o} f_{0}(x)$ solves this problem for each $f_{0} \in \operatorname{Dom}\left(L_{o}\right)$.

2.3. Distributed-order fractional derivatives. There exist many different notions of fractional derivatives. We discuss only two versions of them. One defines the Caputo (or Caputo-Dzhrbashyan) fractional derivative of order $\beta, \beta \in(0,1)$, for a (sufficiently good) function $u$ by

$$
\frac{\partial^{\beta}}{\partial t^{\beta}} u(t):=\frac{1}{\Gamma(1-\beta)} \int_{0}^{t} \frac{u^{\prime}(r)}{(t-r)^{\beta}} d r,
$$

where $\Gamma$ is the Euler's Gamma-function. Let $U$ be the Laplace transform of $u$, i.e. $U(s):=\int_{0}^{\infty} e^{-s t} u(t) d t$. Then the Laplace transform of the Caputo derivative $\frac{\partial^{\beta}}{\partial t^{\beta}} u$

\footnotetext{
${ }^{3}$ Such problems are discussed, e.g., in 29 , see also 38 for the stationary case.
} 
of $u$ can be calculated as follows:

$$
\int_{0}^{\infty} e^{-s t} \frac{\partial^{\beta}}{\partial t^{\beta}} u(t) d t=s^{\beta} U(s)-s^{\beta-1} u(0+) .
$$

The Riemann-Liouville fractional derivative of order $\beta, \beta \in(0,1)$, for a (sufficiently good) function $u$ is defined by

$$
\left(\frac{d}{d t}\right)^{\beta} u(t):=\frac{1}{\Gamma(1-\beta)} \frac{d}{d t} \int_{0}^{t} \frac{u(r)}{(t-r)^{\beta}} d r .
$$

Then the Laplace transform of the Riemann-Liouville derivative $\left(\frac{d}{d t}\right)^{\beta} u$ of $u$ can be calculated as follows:

$$
\int_{0}^{\infty} e^{-s t}\left(\frac{d}{d t}\right)^{\beta} u(t) d t=s^{\beta} U(s) .
$$

Comparing both Laplace transforms and taking into account that the Laplace transform of $t^{-\beta}$ is $s^{\beta-1} \Gamma(1-\beta)$, one sees that if $u$ is absolutely continuous on bounded intervals then the Riemann-Liouville and Caputo derivatives of $u$ are related by

$$
\frac{\partial^{\beta}}{\partial t^{\beta}} u(t)=\left(\frac{d}{d t}\right)^{\beta} u(t)-\frac{t^{-\beta} u(0+)}{\Gamma(1-\beta)} .
$$

The Riemann-Liouville fractional derivative is more general since it does not require the first derivative of $u$ to exist. Therefore, one may adopt the right hand side of the formula (77) to define the Caputo derivative. The further generalization is to consider the so-called distributed order fractional derivative $\mathcal{D}^{\mu}$ with the order $\mu$ determined by a finite Borel measure $\mu$ defined on the interval $(0,1)$ and such that $\mu(0,1)>0$ (cf. [40, 43, 46, 87]):

$$
\mathcal{D}^{\mu} u(t):=\int_{0}^{1} \frac{\partial^{\beta}}{\partial t^{\beta}} u(t) \mu(d \beta)=\int_{0}^{1}\left[\left(\frac{d}{d t}\right)^{\beta} u(t)-\frac{t^{-\beta} u(0)}{\Gamma(1-\beta)}\right] \mu(d \beta) .
$$

If $\mu$ is the Dirac delta-measure $\delta_{\beta_{0}}$ concentrated at a point $\beta_{0} \in(0,1)$, we return to Caputo fractional derivative of $\beta_{0}$-th order.

\section{Chernoff approximation of Semigroups Generated By some killed FELLER PROCESSES}

3.1. General approach. Let $X=C_{0}\left(\mathbb{R}^{d}\right)$. Let $\left(\xi_{t}\right)_{t \geq 0}$ be a doubly Feller process on $\mathbb{R}^{d},\left(T_{t}\right)_{t \geq 0}$ be the corresponding (doubly Feller) semigroup and $(L, \operatorname{Dom}(L)$ ) be its Feller generator. Let a family $(F(t))_{t \geq 0}$ of bounded linear operators on $X$ be Chernoff equivalent 4 to the semigroup $\left(T_{t}\right)_{t \geq 0}$. Therefore, $\|F(t)\| \leq e^{k t}$ for some $k \in \mathbb{R}$ and all $t \geq 0$, and there exists a core $D$ for the operator $(L, \operatorname{Dom}(L))$ such that $\lim _{t \rightarrow 0}\left\|\frac{F(t) \varphi-\varphi}{t}-L \varphi\right\|_{X}=0$ for all $\varphi \in D$. Let us fix this core $D$. Let $G \subset \mathbb{R}^{d}$ be a bounded regular domain. Let $\left(T_{t}^{o}\right)_{t \geq 0}$ be the strongly continuous semigroup on $Y:=C_{0}(G)$ generated by the killed Feller process $\left(\xi_{t}^{o}\right)_{t \geq 0}$ on $G$. Let $\left(L_{o}, \operatorname{Dom}\left(L_{o}\right)\right)$ be the Feller generator of $\left(T_{t}^{o}\right)_{t \geq 0}$. Our aim is to construct a family $\left(F_{o}(t)\right)_{t \geq 0}$ of bounded linear operators on $Y$ which is Chernoff equivalent to $\left(T_{t}^{o}\right)_{t \geq 0}$. The family $\left(F_{o}(t)\right)_{t \geq 0}$ will be constructed by a proper modification of the family $(F(t))_{t \geq 0}$ which is Chernoff equivalent to the semigroup $\left(T_{t}\right)_{t \geq 0}$ on $X$. To this aim, we need some preparations.

\footnotetext{
${ }^{4}$ Note, that some families $(F(t))_{t \geq 0}$, which are Chernoff equivalent to certain Feller semigroups, are constructed, e.g., in [16 21, 22].
} 
Assumption 3.1. We assume that there exists a set $D_{o} \subset \operatorname{Dom}\left(L_{o}\right) \cap C_{b}^{2}(G)$ and a mapping $\mathcal{E}: Y \rightarrow C_{c}\left(\mathbb{R}^{d}\right) \subset X$ such that

(i) $D_{o}$ is a core for $L_{o}$;

(ii) $\left.\mathcal{E}(\varphi)\right|_{\bar{G}}=\varphi$ for all $\varphi \in Y$;

(iii) the mapping $\mathcal{E}$ is linear;

(iv) the mapping $\mathcal{E}$ preserves the supremum norm, i.e. $\|\varphi\|_{Y}=\|\mathcal{E}(\varphi)\|_{X}$ for all $\varphi \in Y$

(v) $\mathcal{E}: D_{o} \rightarrow D$, where $D$ is a fixed core for $(L, \operatorname{Dom}(L))$;

(vi) $L(\mathcal{E}(\varphi))(x)=L_{o} \varphi(x)$ for each $\varphi \in D_{o}$ and each $x \in G$.

Remark 3.1. The space $Y$ can be naturally embedded into $X$ by assigning to each $\varphi \in Y$ zero values outside the domain $G$. However, such embedding produces from smooth functions in $G$ only continuous functions in $\mathbb{R}^{d}$. This may violate the requirement (v) of Assumption 3.1. Note that $\operatorname{Dom}\left(L_{o}\right)$ typically contains functions whose zero extensions do not belong to $\operatorname{Dom}(L)$. Moreover, there is no reason to expect the existence of a core $D_{o}$ such that the zero extensions of its elements belong to $\operatorname{Dom}(L)$. In particular, the sets of sufficiently smooth functions with compact supports in $G$ can not serve as a core even for the Laplacian $\Delta$ in $Y 5$ ! Indeed, assume that there exists a core $D_{o} \subset C_{c}(G)$ for $(\Delta, \operatorname{Dom}(\Delta))$ in $Y$. Then for each $\varphi \in \operatorname{Dom}(\Delta)$ there exists a sequence $\left(\varphi_{n}\right)_{n \in \mathbb{N}} \subset D_{o}$ such that $\left\|\varphi_{n}-\varphi\right\|_{Y} \rightarrow 0$ and $\left\|\Delta \varphi_{n}-\Delta \varphi\right\|_{Y} \rightarrow 0$ as $n \rightarrow \infty$. By Corollary 3.1.21 (i), (ii) and Remark 2.1.5 in [42, $\operatorname{Dom}(\Delta)$ is continuously embedded in $C^{1}(\bar{G})$. Hence $\left\|\frac{\partial \varphi_{n}}{\partial x_{i}}-\frac{\partial \varphi}{\partial x_{i}}\right\|_{Y} \rightarrow 0$ as $n \rightarrow \infty$ for all $i=1, \ldots, d$. Therefore, $\left.\nabla \varphi\right|_{\partial G}=0$ for each $\varphi \in \operatorname{Dom}(\Delta)$. This is however wrong since, e.g., the function $\varphi(x):=\sin x$ belongs to $\operatorname{Dom}(\Delta)=\operatorname{Dom}\left(\frac{d^{2}}{d x^{2}}\right)$ for $G:=(0, \pi)$ and $\frac{d \varphi}{d x}(x)=\cos x$ is not equal to zero on $\partial G$.

Remark 3.2. Let the generator $L$ of a doubly Feller semigroup $\left(T_{t}\right)_{t \geq 0}$ be given for each $\varphi \in C_{0}^{2}\left(\mathbb{R}^{d}\right)$ by

$$
L \varphi(x)=-C(x) \varphi(x)-B(x) \cdot \nabla \varphi(x)+\operatorname{tr}(A(x) \operatorname{Hess} \varphi(x)),
$$

where the coefficients $A, B$ and $C$ are of the class $6 C_{b}^{2, \alpha}\left(\mathbb{R}^{d}\right)$ for some $\alpha \in(0,1)$. Let there exist $a_{0}, A_{0} \in \mathbb{R}$ such that

$$
0<a_{0} \leq A_{0}<\infty \quad \text { and } \quad a_{0}|z|^{2} \leq z \cdot A(x) z \leq A_{0}|z|^{2} \quad \text { for all } x, z \in \mathbb{R}^{d} .
$$

Assume that the coefficients $A, B, C$ are such that the set $C_{c}^{2, \alpha}\left(\mathbb{R}^{d}\right)$ is a core for the generator $L$ in $X$, and let $D:=C_{c}^{2, \alpha}\left(\mathbb{R}^{d}\right)$. Consider $D_{o}:=\left\{\varphi \in C^{2, \alpha}(G): \varphi, L \varphi \in Y\right\}$. If the boundary $\partial G$ is of the class $C^{4, \alpha}$ then there exists a strongly continuous semigroup $\left(T_{t}^{o}\right)_{t \geq 0}$ on $Y$ generated by the closure of $\left(L, D_{o}\right)$ in $Y$ and Assumption 3.1 is fulfilled due to Thm. 2.2 and Thm. 3.4 in [5].

Remark 3.3. Let now the generator $L$ of a Feller semigroup $\left(T_{t}\right)_{t \geq 0}$ on $X$ be such that $C_{c}^{\infty}\left(\mathbb{R}^{d}\right) \subset \operatorname{Dom}(L)$ and $L \varphi$ is given for each $\varphi \in C_{0}^{2}\left(\mathbb{R}^{d}\right)$ by formula (3). Consider $L$ as the sum $L:=L_{1}+L_{2}$, where $L_{1}$ is the differential operator given by

\footnotetext{
${ }^{5}$ This fact together with its proof have been communicated to the author by Professor Alessandra Lunardi.

${ }^{6}$ Here and in the sequel, $C_{b}\left(\mathbb{R}^{d}\right)$ stands for the space of bounded continuous functions on $\mathbb{R}^{d} ; C_{c}\left(\mathbb{R}^{d}\right)$ stands for the space of continuous functions on $\mathbb{R}^{d}$ with compact support; $C^{0, \alpha}\left(\mathbb{R}^{d}\right)$ stands for Hölder continuous functions on $\mathbb{R}^{d}$ with exponent $\alpha \in(0,1] ; C_{b}^{m}\left(\mathbb{R}^{d}\right)=$ $\left\{\varphi \in C^{m}\left(\mathbb{R}^{d}\right): \partial^{\beta} \varphi \in C_{b}\left(\mathbb{R}^{d}\right),|\beta| \leq m\right\} ; C^{m, \alpha}\left(\mathbb{R}^{d}\right)=\left\{\varphi \in C^{m}\left(\mathbb{R}^{d}\right): \partial^{\beta} \varphi \in C^{0, \alpha}\left(\mathbb{R}^{d}\right),|\beta|=m\right\} ;$ $C_{b}^{m, \alpha}\left(\mathbb{R}^{d}\right)=C^{m, \alpha}\left(\mathbb{R}^{d}\right) \cap C_{b}^{m}\left(\mathbb{R}^{d}\right) ; C_{c}^{m, \alpha}\left(\mathbb{R}^{d}\right)=C^{m, \alpha}\left(\mathbb{R}^{d}\right) \cap C_{c}\left(\mathbb{R}^{d}\right)$.
} 
formula (8) and $L_{2}$ is the integral part

$$
L_{2} \varphi(x):=\int_{y \neq 0}\left(\varphi(x+y)-\varphi(x)-\frac{y \cdot \nabla \varphi(x)}{1+|y|^{2}}\right) N(x, d y), \quad \forall \varphi \in C_{0}^{2}\left(\mathbb{R}^{d}\right), \forall x \in \mathbb{R}^{d} .
$$

Let the coefficients $A, B$ and $C$ be again of the class $C_{b}^{2, \alpha}\left(\mathbb{R}^{d}\right)$ for some $\alpha \in(0,1)$. And let there exist $a_{0}, A_{0} \in \mathbb{R}$ such that (9) holds. Assume that the closure of $\left(L_{1}, C_{c}^{2, \alpha}\left(\mathbb{R}^{d}\right)\right)$ generates a strongly continuous contraction semigroup on $X$. Choose the core $D:=C_{c}^{2, \alpha}\left(\mathbb{R}^{d}\right) \subset X$ for the generator $L_{1}$. Let the boundary $\partial G$ be of the class $C^{4, \alpha}$ for some $\alpha \in(0,1)$. Then, as in Remark 3.2. there exists an extension $\mathcal{E}$ satisfying Assumption 3.1 (ii)-(vi) with respect to $L_{1}$. Let $U \subset \mathbb{R}^{d}$ be another bounded domain such that $G \subset U$. One may consider, e.g., $U \equiv U_{\varepsilon}:=\left\{x \in \mathbb{R}^{d}: \operatorname{dist}(x, G)<\varepsilon\right\}$ for some small constant $\varepsilon>0$. Multiplying the extension $\mathcal{E}$ with a proper cut-off function, one obtains another extension $\mathcal{E}_{U}$, satisfying Assumption 3.1 (ii)-(vi) with respect to $L_{1}$ and the condition $\mathcal{E}_{U}: Y \rightarrow C_{c}(U)$. Assume that $N(x, d y)$ is such that $\left(L_{2}, D\right)$ is $L_{1}$-bounded 7 and the closure of $(L, D), L=L_{1}+L_{2}$, generates a doubly Feller semigroup on $X$. Let there exist a core $D_{o} \subset\left\{\varphi \in C^{2, \alpha}(G): \varphi, L_{1} \varphi \in Y\right\}$ for the corresponding killed generator $\left(L_{o}, \operatorname{Dom}\left(L_{o}\right)\right)$. Then the extension $\mathcal{E}_{U}$ satisfies Assumption 3.1 (ii)-(v) with respect to $L, D$ and $D_{o}$. Since $L$ is a non-local operator, Assumption 3.1 (vi) is not fulfilled automatically. And, for each $\varphi \in D_{o}$ and each $x \in G$, we have

$$
\begin{aligned}
L\left(\mathcal{E}_{U}(\varphi)\right)(x)-L_{o} \varphi(x) & =\int_{y \neq 0}\left(\mathcal{E}_{U}(\varphi)(x+y)-\varphi(x+y)\right) N(x, d y) \\
& =\int_{y \in(-x+U \backslash \bar{G})} \mathcal{E}_{U}(\varphi)(x+y) N(x, d y) .
\end{aligned}
$$

Let $L_{2}$ satisfy the additional condition: there exists a bounded domain $U \supset G$ such that for each $x \in G$ holds

$$
\int_{y \in(-x+U \backslash \bar{G})} N(x, d y)=0 .
$$

Then the extension $\mathcal{E}_{U}$ satisfies Assumption 3.1. The condition (10) actually means that the process $\left(\xi_{t}\right)_{t \geq 0}$ is allowed to leave the domain $G$ either continuously, or by a sufficiently large jump which brings the process even out of $U$. Note that if $N(x, d y)$ corresponds to censored processes (i.e., $N(x, d y)$ satisfies (5) ), then the condition (10) is fulfilled. The condition (10) is also fulfilled if, e.g., $\operatorname{supp} N(x, \cdot) \subset$ $\mathbb{R}^{d} \backslash K$ for all $x \in G$ and some compact $K$ such that $\cup_{x \in G}(-x+U \backslash \bar{G}) \subset K$. One can take as $K$, e.g., a ball $B_{R}\left(x_{0}\right)$ such that its center $x_{0} \in G$ and its raduius $R>2 \operatorname{diam} U$.

Consider now a continuous monotone function $s:(0, \infty) \rightarrow(0, \infty)$ such that

$$
\lim _{t \rightarrow 0} \frac{s(t)}{t}=0 .
$$

Define the set $G_{s(t)} \subset G$ by

$$
G_{s(t)}:=\{x \in G: \operatorname{dist}(x, \partial G)>s(t)\} .
$$

Let $\left(\phi_{s(t)}\right)_{t>0}$ be a family of functions $\phi_{s(t)}: \mathbb{R}^{d} \rightarrow[0,1]$ such that all $\phi_{s(t)} \in C_{c}^{\infty}(G)$, we have $\phi_{s(t)}(x)=1, \forall x \in G_{s(t)}, \forall t>0$, and $\lim _{t \rightarrow t^{*}}\left\|\phi_{s(t)}-\phi_{s\left(t^{*}\right)}\right\|_{X}=0$ for each $t^{*}>0$. Note that functions $\phi_{s(t)}$ converge poitwise to the indicator $1_{G}$ of the

\footnotetext{
${ }^{7}$ Note that the fractional Laplacian $-(-\Delta)^{\alpha / 2}$ is $\Delta$-bounded for all $\alpha \in(0,2)$.
} 
domain $G$ when $t \rightarrow 0$. Consider the family $\left(F_{o}(t)\right)_{t \geq 0}$ of operators on $Y$ defined by $F_{o}(0):=$ Id and for each $t>0$, each $\varphi \in Y$ and each $x \in G$

$$
F_{o}(t) \varphi(x):=\phi_{s(t)}(x)[F(t) \mathcal{E}(\varphi)](x)
$$

where the given family $(F(t))_{t \geq 0}$ is Chernoff equivalent to the semigroup $\left(T_{t}\right)_{t \geq 0}$ on $X$ generated by $(L, \operatorname{Dom}(L))$ and $F^{\prime}(0) \varphi=L \varphi$ for all $\varphi \in D$.

Lemma 3.1. The family $\left(F_{o}(t)\right)_{t \geq 0}$ acts on $Y$ and $\left\|F_{o}(t)\right\| \leq\|F(t)\|$. If the family $(F(t))_{t \geq 0}$ is strongly continuous on $X$ then the family $\left(F_{o}(t)\right)_{t \geq 0}$ is strongly continuous on $Y$.

Proof. The family $\left(F_{o}(t)\right)_{t \geq 0}$ acts on $Y$ since, if $\varphi \in Y=C_{0}(G)$, then $\mathcal{E}(\varphi) \epsilon$ $C_{c}\left(\mathbb{R}^{d}\right) \subset C_{\infty}\left(\mathbb{R}^{d}\right)=X, F(t) \mathcal{E}(\varphi) \in X$ and $\phi_{s(t)}[F(t) \mathcal{E}(\varphi)] \in Y$. Moreover,

$$
\begin{aligned}
\left\|F_{0}(t) \varphi\right\|_{Y} & =\sup _{x \in G}\left|\phi_{s(t)}(x)[F(t) \mathcal{E}(\varphi)](x)\right| \\
& \leq\|F(t) \mathcal{E}(\varphi)\|_{X} \leq\|F(t)\|\|\mathcal{E}(\varphi)\|_{X}=\|F(t)\|\|\varphi\|_{Y} .
\end{aligned}
$$

Let us show the strong continuity of the family $\left(F_{o}(t)\right)_{t \geq 0}$ under assumption that the family $(F(t))_{t \geq 0}$ is strongly continuous on $X$. First, for each $\varphi \in Y$

$$
\begin{aligned}
\lim _{t \rightarrow 0} \| F_{o}(t) & \varphi-\varphi \|_{Y}=\limsup _{t \rightarrow 0} \sup _{x \in G}\left|\phi_{s(t)}(x)[F(t) \mathcal{E}(\varphi)](x)-\varphi(x)\right| \\
& =\lim _{t \rightarrow 0} \sup _{x \in G}\left|\phi_{s(t)}(x)([F(t) \mathcal{E}(\varphi)](x)-\mathcal{E}(\varphi)(x))+\varphi(x)\left[\phi_{s(t)}(x)-1\right]\right| \\
& \leq \lim _{t \rightarrow 0}\|F(t) \mathcal{E}(\varphi)-\mathcal{E}(\varphi)\|_{X}+\lim _{t \rightarrow 0} \sup _{x \in \overline{G \backslash G(t)}}|\varphi(x)| \\
& =0
\end{aligned}
$$

due to strong continuity at zero of the family $(F(t))_{t \geq 0}$ on $X$ and uniform continuity of $\varphi$ on the compact $\overline{G \backslash G_{s(t)}}$. Second, for each $t^{*}>0$ and each $\varphi \in Y$

$$
\begin{aligned}
& \lim _{t \rightarrow t^{*}}\left\|F_{o}(t) \varphi-F_{o}\left(t^{*}\right) \varphi\right\|_{Y} \\
& =\lim _{t \rightarrow t^{*}} \sup _{x \in G}\left|\phi_{s(t)}(x)[F(t) \mathcal{E}(\varphi)](x)-\phi_{s\left(t^{*}\right)}(x)\left[F\left(t^{*}\right) \mathcal{E}(\varphi)\right](x)\right| \\
& =\lim _{t \rightarrow t^{*}} \sup _{x \in G} \mid \phi_{s(t)}(x)\left([F(t) \mathcal{E}(\varphi)](x)-\left[F\left(t^{*}\right) \mathcal{E}(\varphi)\right](x)\right)+ \\
& \quad+\left(\phi_{s(t)}(x)-\phi_{s\left(t^{*}\right)}(x)\right)\left[F\left(t^{*}\right) \mathcal{E}(\varphi)\right](x) \mid \\
& \leq \lim _{t \rightarrow t^{*}}\left\|\phi_{s(t)}\right\|_{Y} \cdot\left\|[F(t) \mathcal{E}(\varphi)]-\left[F\left(t^{*}\right) \mathcal{E}(\varphi)\right]\right\|_{X}+\left\|F\left(t^{*}\right) E(\varphi)\right\|_{X} \cdot\left\|\phi_{s(t)}-\phi_{s\left(t^{*}\right)}\right\|_{Y} \\
& =0
\end{aligned}
$$

due to strong continuity of the family $(F(t))_{t \geq 0}$ on $X$ and propersties of the family $\left(\phi_{s(t)}\right)_{t>0}$. Hence Lemma is proved.

Theorem 3.1. Under Assumption 3.1, the family $\left(F_{o}(t)\right)_{t \geq 0}$ is Chernoff equivalent to the semigroup $\left(T_{t}^{o}\right)_{t \geq 0}$, i.e.

$$
T_{t}^{o} \varphi=\lim _{n \rightarrow \infty}\left[F_{o}(t / n)\right]^{n} \varphi
$$

for each $\varphi \in Y$ locally uniformly with respect to $t \geq 0$.

Proof. Due to Lemma 3.1. we have $\left\|F_{o}(t)\right\| \leq\|F(t)\| \leq e^{k t}$ for some $k \in \mathbb{R}$ and all $t \geq 0$. Hence it is sufficient to show that $\lim _{t \rightarrow 0}\left\|t^{-1}\left(F_{o}(t) \varphi-\varphi\right)-L_{o} \varphi\right\|_{Y}=0$ for all 
$\varphi \in D_{o}$. Due to Assumption 3.1

$$
\begin{aligned}
& \left\|\frac{F_{o}(t) \varphi-\varphi}{t}-L_{o} \varphi\right\|_{Y}=\sup _{x \in G}\left|\frac{\phi_{s(t)}(x)[F(t) \mathcal{E}(\varphi)](x)-\varphi(x)}{t}-L_{o} \varphi(x)\right| \\
& \leq \sup _{x \in G}\left[\left|\phi_{s(t)}(x)\right| \frac{F(t) \mathcal{E}(\varphi)(x)-\mathcal{E}(\varphi)(x)}{t}-L \mathcal{E}(\varphi)(x) \mid\right. \\
& \left.\qquad+\left(|\varphi(x) / t|+\left|L_{o} \varphi(x)\right|\right)\left|1-\phi_{s(t)}(x)\right|\right] \\
& \leq \| \frac{F(t) \mathcal{E}(\varphi)-\mathcal{E}(\varphi)}{t}-\left.L \mathcal{E}(\varphi)\right|_{X}+\frac{\sup }{x \in G \backslash G_{s(t)}}\left(|\varphi(x) / t|+\left|L_{o} \varphi(x)\right|\right) \\
& \rightarrow 0, \quad \text { as } t \rightarrow 0 .
\end{aligned}
$$

Indeed, $\lim _{t \rightarrow 0}\left\|\frac{F(t) \mathcal{E}(\varphi)-\mathcal{E}(\varphi)}{t}-L \mathcal{E}(\varphi)\right\|_{X}=0$ since $\mathcal{E}(\varphi) \in D$ and $F^{\prime}(0)=L$ on $D$ by our assumptions. Further, $\varphi \in D_{o} \subset C_{b}^{2}(G) \cap Y$. Hence $\varphi$ is Lipschitz on $\bar{G}$, i.e. there exists a constant $M>0$ such that the inequality $|\varphi(x)-\varphi(z)| \leq M|x-z|$ holds for all $x, z \in \bar{G}$. Moreover, for each $x \in G \backslash G_{s(t)}$, there exists at least one point $z_{x} \in \partial G$ such that $\operatorname{dist}\left(x, z_{x}\right) \leq s(t)$. Therefore, $|\varphi(x)|=\left|\varphi(x)-\varphi\left(z_{x}\right)\right| \leq M s(t)$ for each $x \in G \backslash G_{s(t)}$. And

$$
\lim _{t \rightarrow 0} \frac{\sup }{x \in \overline{G \backslash G_{s(t)}}} \frac{|\varphi(x)|}{t} \leq \lim _{t \rightarrow 0} M \frac{s(t)}{t}=0 .
$$

Besides, since $\varphi \in D_{o} \subset \operatorname{Dom}\left(L_{o}\right)$, we have $L_{o} \varphi \in Y=C_{0}(G)$. Hence

$$
\lim _{t \rightarrow 0} \sup _{x \in G \backslash G_{s(t)}}\left|L_{o} \varphi(x)\right|=0
$$

due to uniform continuity of the function $L_{o} \varphi$ on compacts $\overline{G \backslash G_{s(t)}}$. Thus, Theorem is proved.

Remark 3.4. Analogues of Theorem 3.1 are also valid in unbounded domains $G \subset$ $\mathbb{R}^{d}$, in domains $G$ of a locally compact metric space $Q$ and in other couples of Banach spaces $X$ and $Y$ (e.g., $X:=L^{p}\left(\mathbb{R}^{d}\right)$ and $\left.Y:=L^{p}(G), p \in[1, \infty)\right)$ under corresponding modifications of Assumption 3.1 and properties of the family $\left(\phi_{s(t)}\right)_{t>0}$, as well as under additional assumption on the existence of the semigroup $\left(T_{t}^{o}\right)_{t \geq 0}$.

3.2. Feynman Formula for semigroups generated by a class of killed Feller processes. Let $\left(T_{t}\right)_{t \geq 0}$ be a doubly Feller semigroup on $X$ whose generator $(L, \operatorname{Dom}(L))$ is such that the set $D:=C_{c}^{\infty}\left(\mathbb{R}^{d}\right)$ is a core for $L$. Hence $L \varphi$ is given by formula (3) for each $\varphi \in C_{0}^{2}\left(\mathbb{R}^{d}\right)$. Assume that the coefficients $A, B, C$ in formula (3) are bounded and continuous. Let there exist $a_{0}, A_{0} \in \mathbb{R}$ such that condition (9) holds. Let the measure $N(x, d y)$ in formula (3) do not depend on $x$, i.e. $N(x, d y):=N(d y)$ for all $x \in \mathbb{R}^{d}$. Let $\left(\eta_{t}\right)_{t \geq 0}$ be the convolution semigroup on $\mathbb{R}^{d}$ corresponding 8 to $N(d y)$. Then, by Thm. 3.1 in [21] and Remark 15 in [19], the following family $(F(t))_{t \geq 0}$ on $X$ is Chernoff equivalent to $\left(T_{t}\right)_{t \geq 0}: F(0)=\mathrm{Id}$ and for all $t>0$, all $\varphi \in X$ and all $x \in \mathbb{R}^{d}$

$$
F(t) \varphi(x):=\frac{e^{-t C(x)}}{\sqrt{(4 \pi t)^{d} \operatorname{det} A(x)}} \int_{\mathbb{R}^{d}} \int_{\mathbb{R}^{d}} e^{-\frac{A^{-1}(x)(z-x+t B(x)+y) \cdot(z-x+t B(x)+y)}{4 t}} \varphi(y) d y \eta_{t}(d z) .
$$

\footnotetext{
${ }^{8}$ I.e. the Fourier transforms $\mathcal{F}\left[\eta_{t}\right]$ of sub-probability measures $\eta_{t}$ for all $t \geq 0$ are given by $\mathcal{F}\left[\eta_{t}\right](x)=(2 \pi)^{-d / 2} e^{-t r(x)}$, where the function $r: \mathbb{R}^{d} \rightarrow \mathbb{C}$ is defined by $r(x):=$ $\int_{\mathbb{R}^{d} \backslash\{0\}}\left(1-e^{i y \cdot x}+\frac{i y \cdot x}{1+|y|^{2}}\right) N(d y)$.
} 
Moreover, the family $(F(t))_{t \geq 0}$ is a strongly continuous family of contractions. Note also that, for $g(x) \equiv 1$, we have $F(t) g(x)=\exp \{-t C(x)\} \leq 1$ for all $x \in \mathbb{R}^{d}$.

Let $G \subset \mathbb{R}^{d}$ be a regular bounded domain. Consider the corresponding Feller semigroup $\left(T_{t}^{o}\right)_{t \geq 0}$ on $Y$. Let Assumption 3.1 be fulfilled for some core $D_{o}$ of the generator of $\left(T_{t}^{o}\right)_{t \geq 0}$ and for some extension $\mathcal{E}: Y \rightarrow X$ with respect to $D_{o}$ and $D:=C_{c}^{\infty}\left(\mathbb{R}^{d}\right)$. Then, by Theorem 3.1, the family $\left(F_{o}(t)\right)_{t \geq 0}$, constructed from the family $(F(t))_{t \geq 0}$ in (12) through the formula (11), is Chernoff equivalent to the semigroup $\left(T_{t}^{o}\right)_{t \geq 0}$. Hence $F_{o}(0)=\mathrm{Id}$ and for all $t>0$ and all $\varphi \in Y$

$$
\begin{aligned}
& F_{o}(t) \varphi(x):=\frac{\phi_{s(t)}(x) e^{-t C(x)}}{\sqrt{(4 \pi t)^{d} \operatorname{det} A(x)}} \times \\
& \times \int_{\mathbb{R}^{d}} \int_{\mathbb{R}^{d}} \exp \left\{-\frac{A^{-1}(x)(z-x+t B(x)+y) \cdot(z-x+t B(x)+y)}{4 t}\right\} \mathcal{E}(\varphi)(y) d y \eta_{t}(d z) .
\end{aligned}
$$

Therefore, we have uniformly with respect to $x_{0} \in G$ and uniformly with respect to $t \in\left(0, t^{*}\right]$ for all $t^{*}>0$

$$
\begin{aligned}
T_{t}^{o} \varphi\left(x_{0}\right)=\lim _{n \rightarrow \infty} F_{o}^{n}(t / n) \varphi\left(x_{0}\right)= & \lim _{n \rightarrow \infty} \int_{\mathbb{R}^{d}} \int_{\mathbb{R}^{d}} \ldots \int_{\mathbb{R}^{d}} \int_{\mathbb{R}^{d}}\left(\prod_{k=1}^{n} \phi_{s(t / n)}\left(x_{k-1}\right)\right) \mathcal{E}(\varphi)\left(x_{n}\right) \times \\
& \times \Psi_{t, n}^{x_{0}}\left(x_{1}, \ldots, z_{n}\right) d x_{n} \eta_{t / n}\left(d z_{n}\right) \cdots d x_{1} \eta_{t / n}\left(d z_{1}\right),
\end{aligned}
$$

where

$$
\begin{aligned}
& \Psi_{t, n}^{x_{0}}\left(x_{1}, \ldots, z_{n}\right):=\left(\prod_{k=1}^{n}(4 \pi t / n)^{-d / 2}\left(\operatorname{det} A\left(x_{k-1}\right)\right)^{-1 / 2}\right) \exp \left\{-\frac{t}{n} \sum_{k=1}^{n} C\left(x_{k-1}\right)\right\} \times \\
& \times \exp \left\{-\sum_{k=1}^{n} \frac{A^{-1}\left(x_{k-1}\right)\left(z_{k}-x_{k-1}+t B\left(x_{k-1}\right)+x_{k}\right) \cdot\left(z_{k}-x_{k-1}+t B\left(x_{k-1}\right)+x_{k}\right)}{4 t / n}\right\} .
\end{aligned}
$$

Since all $\phi_{s(t)}$ are smooth functions with compact supports in $G$ and $\mathcal{E}(\varphi)$ is a continuous function with compact support $K:=\operatorname{supp} \mathcal{E}(\varphi)$, the $2 n$-fold iterated integrals over $\mathbb{R}^{d}$ in (14) coincide with the following $2 n$-fold multiple integral

$$
\begin{aligned}
\Phi_{n}^{\varphi}\left(t, x_{0}\right):=\int_{G^{n-1} \times K \times \mathbb{R}^{n d}} & \left(\prod_{k=1}^{n} \phi_{s(t / n)}\left(x_{k-1}\right)\right) \mathcal{E}(\varphi)\left(x_{n}\right) \times \\
& \times \Psi_{t, n}^{x_{0}}\left(x_{1}, \ldots, z_{n}\right) d x_{1} \cdots d x_{n} \eta_{t / n}\left(d z_{1}\right) \cdots \eta_{t / n}\left(d z_{n}\right) .
\end{aligned}
$$

Consider also

$$
\Theta_{n}^{\varphi}\left(t, x_{0}\right):=\int_{G^{n} \times \mathbb{R}^{n d}} \varphi\left(x_{n}\right) \Psi_{t, n}^{x_{0}}\left(x_{1}, \ldots, z_{n}\right) d x_{1} \cdots d x_{n} \eta_{t / n}\left(d z_{1}\right) \cdots \eta_{t / n}\left(d z_{n}\right) .
$$

Let us show that for all $t>0$ and all $x_{0} \in G$ holds

$$
T_{t}^{o} \varphi\left(x_{0}\right)=\lim _{n \rightarrow \infty} \Theta_{n}^{\varphi}\left(t, x_{0}\right) .
$$

And the convergence in (15) is locally uniform with respect to $x_{0} \in G$ and uniform with respect to $t \in\left(0, t^{*}\right]$ for all $t^{*}>0$. So, consider a number $t^{*}>0$ and a compact $\Upsilon \subset G$. Let $x_{0} \in \Upsilon$ and $t \in\left(0, t^{*}\right]$. Then

$$
\begin{array}{r}
\left|\Phi_{n}^{\varphi}\left(t, x_{0}\right)-\Theta_{n}^{\varphi}\left(t, x_{0}\right)\right| \leq \int_{G^{n-1} \times K \times \mathbb{R}^{n d}}\left(\prod_{k=1}^{n}\left|\phi_{s(t / n)}\left(x_{k-1}\right)-1_{G}\left(x_{k-1}\right)\right|\right)\left|\mathcal{E}(\varphi)\left(x_{n}\right)\right| \times \\
\times \Psi_{t, n}^{x_{0}}\left(x_{1}, \ldots, z_{n}\right) d x_{1} \cdots d x_{n} \eta_{t / n}\left(d z_{1}\right) \cdots \eta_{t / n}\left(d z_{n}\right) \\
+\int_{G^{n-1} \times \mathbb{R}^{n d}}\left(\int_{K \backslash G}\left|\mathcal{E}(\varphi)\left(x_{n}\right)\right| \Psi_{t, n}^{x_{0}}\left(x_{1}, \ldots, z_{n}\right) d x_{n}\right) d x_{1} \cdots d x_{n-1} \eta_{t / n}\left(d z_{1}\right) \cdots \eta_{t / n}\left(d z_{n}\right) .
\end{array}
$$


Let us estimate each of the summands separately. Denote the first summand by $I_{n}^{\varphi}\left(t, x_{0}\right)$ and the second by $J_{n}^{\varphi}\left(t, x_{0}\right)$. We have with $g(x) \equiv 1$

$$
\begin{aligned}
& I_{n}^{\varphi}\left(t, x_{0}\right) \\
& \leq\|\varphi\|_{Y}\left|\phi_{s(t / n)}\left(x_{0}\right)-1_{G}\left(x_{0}\right)\right| \int_{\mathbb{R}^{d}} \ldots \int_{\mathbb{R}^{d}} \Psi_{t, n}^{x_{0}}\left(x_{1}, \ldots, z_{n}\right) d x_{n} \eta_{t / n}\left(d z_{n}\right) \cdots d x_{1} \eta_{t / n}\left(d z_{1}\right) \\
& =\|\varphi\|_{Y}\left|\phi_{s(t / n)}\left(x_{0}\right)-1_{G}\left(x_{0}\right)\right|\left(F^{n}(t / n) g\left(x_{0}\right)\right) \leq\|\varphi\|_{Y}\left|\phi_{s(t / n)}\left(x_{0}\right)-1_{G}\left(x_{0}\right)\right| .
\end{aligned}
$$

By the construction of the sets $G_{s(t)}$, there exists $N \in \mathbb{N}$ such that $\Upsilon \subset G_{s\left(t^{*} / N\right)}$. Hence for all $n \geq N, x_{0} \in \Upsilon, t \in\left(0, t^{*}\right]$ holds $\left|\phi_{s(t / n)}\left(x_{0}\right)-1_{G}\left(x_{0}\right)\right|=0$. Therefore,

$$
\lim _{n \rightarrow \infty} I_{n}^{\varphi}\left(t, x_{0}\right)=0 \quad \text { uniformly with respect to } x_{0} \in \Upsilon, t \in\left(0, t^{*}\right] .
$$

Consider now the second summand $J_{n}^{\varphi}\left(t, x_{0}\right)$. Due to condition (9), we have for all $x \in \bar{G}, y \in K, z \in \mathbb{R}^{d}, t \in\left(0, t^{*}\right]$ and $n \in \mathbb{N}$

$$
\begin{aligned}
& \frac{e^{-t C(x) / n}}{\sqrt{(4 \pi t / n)^{d} \operatorname{det} A(x)}} \exp \left\{-\frac{A^{-1}(x)(z-x+t B(x) / n+y) \cdot(z-x+t B(x) / n+y)}{4 t / n}\right\} \\
& \leq M\left(A_{0} / a_{0}\right)^{d / 2}\left(4 A_{0} \pi t / n\right)^{-d / 2} \exp \left\{-\frac{|x-y|^{2}}{4 A_{0} t / n}\right\}
\end{aligned}
$$

where

$$
M:=\sup _{x \in \bar{G}, y \in K, z \in \mathbb{R}^{d}, t \in\left(0, t^{*}\right], n \in \mathbb{N}} \exp \left\{-\frac{|z+t B(x) / n|^{2}+2(z+t B(x) / n) \cdot(y-x)}{4 A_{0} t / n}\right\}<\infty .
$$

Therefore, with $c:=M\left(A_{0} / a_{0}\right)^{d / 2}$ and $p_{A_{0}}(t, x, y):=\left(4 A_{0} \pi t\right)^{-d / 2} \exp \left\{-\frac{|x-y|^{2}}{4 A_{0} t}\right\}$

$$
\begin{aligned}
J_{n}^{\varphi}\left(t, x_{0}\right) & \leq c\left(\sup _{x \in \bar{G}} \int_{K \backslash G} p_{A_{0}}(t / n, x, y)|\mathcal{E}(\varphi)(y)| d y\right)\left(F^{n-1}(t / n) g\left(x_{0}\right)\right) \\
& \leq c\left(\sup _{x \in \bar{G}_{K \backslash G}} \int_{A_{0}} p_{A_{0}}(t / n, x, y)|\mathcal{E}(\varphi)(y)| d y\right) .
\end{aligned}
$$

Denote by $G^{\delta}$ the $\delta$-neighborhood of $G$ in $\mathbb{R}^{d}$, i.e. $G^{\delta}:=\left\{x \in \mathbb{R}^{d}: \operatorname{dist}(x, G)<\delta\right\}$, $\delta>0$. Fix any $\varepsilon>0$. Since $\mathcal{E}(\varphi)$ is a continuous function on $\mathbb{R}^{d}$ which equals zero on $\partial G$, there exists $\delta>0$ such that $|\mathcal{E}(\varphi)| \leq \varepsilon / 2$ on $G^{\delta} \backslash G$. Hence

$$
\begin{aligned}
& \sup _{x \in \bar{G}} \int_{K \backslash G} p_{A_{0}}(t / n, x, y)|\mathcal{E}(\varphi)(y)| d y \leq \sup _{x \in \bar{G}_{G^{\delta} \backslash G}} p_{A_{0}}(t / n, x, y)|\mathcal{E}(\varphi)(y)| d y \\
& +\sup _{x \in \bar{G}_{K \backslash G^{\delta}}} \int_{A_{0}}(t / n, x, y)|\mathcal{E}(\varphi)(y)| d y \leq \frac{\varepsilon}{2}+\|\varphi\|_{Y} \sup _{x \in \bar{G}_{K} \backslash G^{\delta}} p_{A_{0}}(t / n, x, y) d y .
\end{aligned}
$$

Due to Gaussian fall off of $p_{A_{0}}$ there exists $N \in \mathbb{N}$ such that for all $n \geq N$ and all $t \in\left(0, t^{*}\right]$ holds

$$
\|\varphi\|_{Y} \sup _{x \in \bar{G}} \int_{K \backslash G^{\delta}} p_{A_{0}}(t / n, x, y) d y \leq \frac{\varepsilon}{2} .
$$

Consequently, since $\varepsilon>0$ has been chosen arbitrary,

$$
\lim _{n \rightarrow \infty} J_{n}^{\varphi}\left(t, x_{0}\right)=0 \quad \text { uniformly with respect to } x_{0} \in \Upsilon, t \in\left(0, t^{*}\right] .
$$

Therefore, the following statement is proved. 
Proposition 3.1. Under all assumptions of this Subsection, the following Feynman formula holds for the semigroup $\left(T_{t}^{o}\right)_{t \geq 0}$ :

$$
\begin{aligned}
& T_{t}^{o} \varphi\left(x_{0}\right)=\lim _{n \rightarrow \infty} \Theta_{n}^{\varphi}\left(t, x_{0}\right)=\lim _{n \rightarrow \infty} \int_{G^{n} \times \mathbb{R}^{n d}} \varphi\left(x_{n}\right)\left(\prod_{k=1}^{n}(4 \pi t / n)^{-d / 2}\left(\operatorname{det} A\left(x_{k-1}\right)\right)^{-1 / 2}\right) \times \\
& \times \exp \left\{-\sum_{k=1}^{n} \frac{A^{-1}\left(x_{k-1}\right)\left(z_{k}-x_{k-1}+t B\left(x_{k-1}\right)+x_{k}\right) \cdot\left(z_{k}-x_{k-1}+t B\left(x_{k-1}\right)+x_{k}\right)}{4 t / n}\right\} \times \\
& \quad \times \exp \left\{-\frac{t}{n} \sum_{k=1}^{n} C\left(x_{k-1}\right)\right\} d x_{1} \cdots d x_{n} \eta_{t / n}\left(d z_{1}\right) \cdots \eta_{t / n}\left(d z_{n}\right), \quad \forall \varphi \in Y, \forall x_{0} \in G .
\end{aligned}
$$

And the convergence in this Feynman formula is locally uniform with respect to $x_{0} \in G$ and uniform with respect to $t \in\left(0, t^{*}\right]$ for all $t^{*}>0$.

As a particular case, we have the following (cf. [18).

Corollary 3.1. Let all the assumptions of this Subsection be fulfilled. Let $N(d y) \equiv$ 0 , i.e. $\eta_{t}=\delta_{0}$ for all $t \geq 0$. Then the family $(F(t))_{t \geq 0}$ given in (12) has the following view: $F(0):=\operatorname{Id}$ and for all $t>0$ and all $\varphi \in X$

$$
\begin{aligned}
F(t) \varphi(x): & =\frac{e^{-t C(x)}}{\sqrt{(4 \pi t)^{d} \operatorname{det} A(x)}} \int_{\mathbb{R}^{d}} e^{-\frac{A^{-1}(x)(x-t B(x)-y) \cdot(x-t B(x)-y)}{4 t}} \varphi(y) d y \\
& \equiv e^{-t C(x)} \int_{\mathbb{R}^{d}} e^{\frac{A^{-1}(x) B(x) \cdot(x-y)}{2}} e^{-t \frac{\left|A^{-1 / 2}(x) B(x)\right|^{2}}{4}} \varphi(y) p_{A}(t, x, y) d y,
\end{aligned}
$$

where for all $x, y \in \mathbb{R}^{d}$

$$
p_{A}(t, x, y):=\frac{1}{\sqrt{(4 \pi t)^{d} \operatorname{det} A(x)}} \exp \left(-\frac{A^{-1}(x)(x-y) \cdot(x-y)}{4 t}\right) .
$$

This family $(F(t))_{t \geq 0}$ is a strongly continuous family of contractions on $X$ which is Chernoff equivalent to the semigroup $\left(T_{t}\right)_{t \geq 0}$. Moreover, the corresponding semigroup $\left(T_{t}^{o}\right)_{t \geq 0}$ can be approximated via the following Feynman formula:

$$
\begin{aligned}
T_{t}^{o} \varphi\left(x_{0}\right)=\lim _{n \rightarrow \infty} \int_{G^{n}} \exp \left(-\frac{t}{n} \sum_{j=1}^{n}\left(C\left(x_{j-1}\right)+\frac{1}{4}\left|A^{-1 / 2}\left(x_{j-1}\right) B\left(x_{j-1}\right)\right|^{2}\right)\right) \\
\quad \times \exp \left(-\frac{1}{2} \sum_{j=1}^{n} A^{-1}\left(x_{j-1}\right) B\left(x_{j-1}\right) \cdot\left(x_{j}-x_{j-1}\right)\right) \varphi\left(x_{n}\right) \\
\quad \times p_{A}\left(t / n, x_{0}, x_{1}\right) \cdots p_{A}\left(t / n, x_{n-1}, x_{n}\right) d x_{1} \ldots d x_{n},
\end{aligned}
$$

for each $\varphi \in Y$, each $x_{0} \in G$ and each $t>0$. The convergence in the Feynman formula (19) is locally uniform with respect to $x_{0} \in G$ and uniform with respect to $t \in\left(0, t^{*}\right]$ for all $t^{*}>0$.

Remark 3.5. It is assumed in this Subsection that $C_{c}^{\infty}\left(\mathbb{R}^{d}\right)$ is a core for $L$. If $L$ is given by formula (8) with continuous and bounded coefficients $A, B, C$, then $C_{c}^{\infty}\left(\mathbb{R}^{d}\right) \subset C_{c}^{2, \alpha}\left(\mathbb{R}^{d}\right) \subset \operatorname{Dom}(L)$ and hence $C_{c}^{2, \alpha}\left(\mathbb{R}^{d}\right)$ is also a core for $L$. Therefore, one may consider $D:=C_{c}^{2, \alpha}\left(\mathbb{R}^{d}\right.$ ) (or any other bigger core for $L$ ) and find corresponding $D_{o}$ and $\mathcal{E}$ such that Assumption 3.1 holds. In principle, the bigger $D$ is chosen, the easier is to find $\mathcal{E}$ satisfying the condition (v) of Assumption 3.1 that $\mathcal{E}\left(D_{o}\right) \subset D$. As it has been shown in [18, the family $(F(t))_{t \geq 0}$ given in (17) satisfies the condition

$$
\lim _{t \rightarrow 0}\left\|\frac{F(t) \varphi-\varphi}{t}-L \varphi\right\|_{X}=0 \quad \text { for all } \varphi \in C_{c}^{2, \alpha}\left(\mathbb{R}^{d}\right), \alpha \in(0,1) .
$$


Therefore, it is sufficient to assume that $C_{c}^{2, \alpha}\left(\mathbb{R}^{d}\right)$ is a core for $L$ in Corollary 3.1

\section{Approximation of solutions and Feynman formulae for TIME-FRACTIONAL FOKKER-PLANCK-KOLMOGOROV EQUATIONS}

4.1. Approximation of solutions for distributed order time-fractional Fokker-Planck-Kolmogorov equations. We are interested now in distributed order time-fractional evolution equations of the form

$$
\mathcal{D}^{\mu} f(t, x)=L f(t, x),
$$

where $\mathcal{D}^{\mu}$ is the distributed order fractional derivative with respect to the time variable $t$ and $L$ is the generator of a strongly continuous semigroup $\left(T_{t}\right)_{t \geq 0}$ on some Banach space $\left(X,\|\cdot\|_{X}\right)$ of functions of the space variable $x$. Equations of such type are called time-fractional Fokker-Planck-Kolmogorov equations and arise in the framework of continuous time random walks (CTRWs) and fractional kinetic theory (32, 54, 50, 91]). As it is shown in papers 57, 36, 52 (see also papers [33, 3, 47, 88, 49] for the case $\left.\mu=\delta_{\beta_{0}}, \beta_{0} \in(0,1)\right)$, such time-fractional Fokker-Planck-Kolmogorov equations are governing equations for stochastic processes which are weak limits of certain sequences or triangular arrays of CTRWs. These limit processes are actually time-changed Lévy processes, where the timechange arises as the first hitting time of level $t>0$ (or, equivalently, as the inverse process) for a mixture of independent stable subordinators with some mixing measure $\mu$.

Recall that a process $\left(D_{t}^{\beta}\right)_{t \geq 0}$ with $\beta \in(0,1)$ is $\beta$-stable subordinator if it is a one-dimensional Lévy proces with almost surely non-decreasing paths such that the corresponding Bernstein function is $f(s):=s^{\beta}$ (see, e.g., Section 3.9 of 39] for the definitions). For a given finite Borel measure $\mu$ with $\operatorname{supp} \mu \in(0,1)$, consider the function $f^{\mu}$ given by

$$
f^{\mu}(s):=\int_{0}^{1} s^{\beta} \mu(d \beta), \quad s>0 .
$$

It is a Bernstein function given by (cf. formula (3.246) of [39]):

$$
f^{\mu}(s)=\int_{0+}^{\infty}\left(1-e^{-t s}\right) m(d t), \quad \text { where } m(d t):=\left(\int_{0}^{1} \frac{\beta t^{-\beta-1}}{\Gamma(1-\beta)} \mu(d \beta)\right) d t .
$$

Let $\left(D_{t}^{\mu}\right)_{t \geq 0}$ be a subordinator corresponding to the Bernstein function $f^{\mu}$. This process represents a mixture of independent stable subordinators with a mixing measure $\mu$. Define now the process $\left(E_{t}^{\mu}\right)_{t \geq 0}$ by

$$
E_{t}^{\mu}:=\inf \left\{\tau \geq 0: D_{\tau}^{\mu}>t\right\}
$$

The process $\left(E_{t}^{\mu}\right)_{t \geq 0}$ is the first hitting time of the level $t$ of the process $\left(D_{\tau}^{\mu}\right)_{\tau \geq 0}$ or, equivalently, the inverse to $\left(D_{t}^{\mu}\right)_{t \geq 0}$. This process $\left(E_{t}^{\mu}\right)_{t \geq 0}$ is sometimes called inverse subordinator. However, note that it is not a Markov process. It is known that $\left(E_{t}^{\mu}\right)_{t \geq 0}$ possesses a marginal density function $p^{\mu}(t, x)$, i.e. $\mathbb{P}\left(E_{t}^{\mu} \in B\right)=$ $\int_{B} p^{\mu}(t, x) d x$ for all $B \in \mathcal{B}(\mathbb{R})$, and $p^{\mu}(t, x)=0$ for all $x<0$. The marginal density function $p^{\mu}(t, x)$ has many nice properties (see Lemma 2.4 and Lemma 2.5 in [35]; for the case $\mu=\delta_{\beta_{0}}, \beta_{0} \in(0,1)$, see also [45, 28] $)$. In particular, $p^{\mu} \in C^{\infty}((0, \infty) \times$ $(0, \infty))$. In the sequel, we need the following simple property of $p^{\mu}$ : 
Lemma 4.1. For each $\varepsilon>0$ and each $T>0$ there exists $R_{T, \varepsilon}>0$ such that for all $t \in[0, T]$ holds

$$
\int_{R_{T, \varepsilon}}^{\infty} p^{\mu}(t, x) d x<\varepsilon .
$$

Proof. Choose arbitrary $\varepsilon>0$ and $T>0$. Consider $R>0$. We have for all $t \in[0, T]$ :

$$
\begin{aligned}
\int_{R}^{\infty} p^{\mu}(t, x) d x & =\mathbb{P}\left(E_{t}^{\mu} \geq R\right)=\mathbb{P}\left(D_{R}^{\mu} \leq t\right) \\
& \leq \mathbb{P}\left(D_{R}^{\mu} \leq T\right)=\mathbb{P}\left(E_{T}^{\mu} \geq R\right)=\int_{R}^{\infty} p^{\mu}(T, x) d x \\
& <\varepsilon
\end{aligned}
$$

for sufficiently large $R$ since $\int_{0}^{\infty} p^{\mu}(T, x) d x=1$.

The marginal density function $p^{\mu}(t, x)$ allows to represent solutions of Cauchy problems for distributed order time-fractional evolution equations of the form (20) in the following way (cf. Thm. 3.2 in [36] and Thm. 4.2 in [52]):

Proposition 4.1. Let $\left(X,\|\cdot\|_{X}\right)$ be a Banach space. Let $(L, \operatorname{Dom}(L))$ be the generator of a uniformly bounded strongly continuous semigroup $\left(T_{t}\right)_{t \geq 0}$ on $X$. Let $f_{0} \in \operatorname{Dom}(L)$. Let $\mu$ be a finite Borel measure with $\operatorname{supp} \mu \in(0,1)$. Then the family of linear operators $\left(\mathcal{T}_{t}\right)_{t \geq 0}$ from $X$ into $X$ given by

$$
\mathcal{T}_{t} \varphi:=\int_{0}^{\infty} T_{\tau} \varphi p^{\mu}(t, \tau) d \tau, \quad \forall \varphi \in X,
$$

is uniformly bounded and strongly analytic in a sectorial region. Furthermore, the family $\left(\mathcal{T}_{t}\right)_{t \geq 0}$ is strongly continuous and the function $f(t):=\mathcal{T}_{t} f_{0}$ is a solution of the Cauchy problem

$$
\begin{aligned}
& \mathcal{D}^{\mu} f(t)=L f(t), \quad t>0, \\
& f(0)=f_{0} .
\end{aligned}
$$

This result shows that solutions of time-fractional evolution equations are a kind of subordination of solutions of the corresponding time-non-fractional evolution equations with respect to "subordinators" $\left(E_{t}^{\mu}\right)_{t \geq 0}$. And respectively, if a timenon-fractional evolution equation is a governing equation for a Markov process then the related time-fractional evolution equation is a governing equation for a (already non-markovian) process which is a "subordination", i.e. a time-change of this Markov process by means of $\left(E_{t}^{\mu}\right)_{t \geq 0}$. The non-Markovity of the resulting process corresponds to the fact that the family $\left(\mathcal{T}_{t}\right)_{t \geq 0}$ is not a semigroup any more. Note also that some other types of time-fractional evolution equations have a similar "subordination-like" structure of solutions (see [68, 55]).

Assume now that the semigroup $\left(T_{t}\right)_{t \geq 0}$ is not known explicitly but is already Chernoff approximated. We have no chances to construct Chernoff approximations for the family $\left(\mathcal{T}_{t}\right)_{t>0}$ which is not a semigroup. Nevertheless, the following is true.

Theorem 4.1. Let $\left(X,\|\cdot\|_{X}\right)$ be a Banach space. Let $(L, \operatorname{Dom}(L))$ be the generator of a strongly continuous contraction semigroup $\left(T_{t}\right)_{t \geq 0}$ on $X$. Let the family

\footnotetext{
${ }^{9}$ This means that $\left\|T_{t}\right\| \leq M$ for some $M>0$ and all $t \geq 0$.
} 
$(F(t))_{t \geq 0}$ of contractions on $X$ be strongly Borel measurabl 10 and Chernoff equivalent to $\left(T_{t}\right)_{t \geq 0}$. Let $f_{0} \in \operatorname{Dom}(L)$. Let $\mu$ be a finite Borel measure with supp $\mu \in(0,1)$ and the family $\left(\mathcal{T}_{t}\right)_{t \geq 0}$ be given by formula (21). Let $f:[0, \infty) \rightarrow X$ be defined via $f(t):=\mathcal{T}_{t} f_{0}$. For each $n \in \mathbb{N}$ define the mappings $f_{n}:[0, \infty) \rightarrow X$ by

$$
f_{n}(t):=\int_{0}^{\infty} F^{n}(\tau / n) f_{0} p^{\mu}(t, \tau) d \tau .
$$

Then it holds locally uniformly with respect to $t \geq 0$ that

$$
\left\|f_{n}(t)-f(t)\right\|_{X} \rightarrow 0, \quad n \rightarrow \infty .
$$

Proof. Take any $T>0$ and any $\varepsilon>0$. Due to Lemma 4.1. there exists $R_{T, \varepsilon}>0$ such that

$$
\int_{R_{T, \varepsilon}}^{\infty} p^{\mu}(t, \tau) d \tau<\varepsilon
$$

for all $t \in[0, T]$. Then it holds for $t \in[0, T]$

$$
\begin{aligned}
& \left\|f_{n}(t)-f(t)\right\|_{X}=\left\|\int_{0}^{\infty} F^{n}(\tau / n) f_{0} p^{\mu}(t, \tau) d \tau-\int_{0}^{\infty} T_{\tau} f_{0} p^{\mu}(t, \tau) d \tau\right\|_{X} \\
& \quad \leq \int_{0}^{\infty}\left\|T_{\tau} f_{0}-F^{n}(\tau / n) f_{0}\right\|_{X} p^{\mu}(t, \tau) d \tau \\
& \quad \leq \int_{0}^{R_{T, \varepsilon}}\left\|T_{\tau} f_{0}-F^{n}(\tau / n) f_{0}\right\|_{X} p^{\mu}(t, \tau) d \tau+\int_{R_{T, \varepsilon}}^{\infty}\left\|T_{\tau} f_{0}-F^{n}(\tau / n) f_{0}\right\|_{X} p^{\mu}(t, \tau) d \tau \\
& \quad \leq \sup _{\tau \in\left[0, R_{T, \varepsilon}\right]}\left\|T_{\tau} f_{0}-F^{n}(\tau / n) f_{0}\right\|_{X} \int_{0}^{R_{T, \varepsilon}} p^{\mu}(t, \tau) d \tau+2 \varepsilon\left\|_{0}\right\|_{X} \\
& \quad \rightarrow 2 \varepsilon\left\|f_{0}\right\|_{X}, \quad n \rightarrow \infty,
\end{aligned}
$$

due to the fact that the convergence in the Chernof theorem is locally uniform with respect to the time variable. Since $\varepsilon>0$ was chosen arbitrary, the statement follows.

Remark 4.1. Consider a time-fractional Fokker-Planck-Kolmogorov equation of the form (20). Assume that the semigroup $\left(T_{t}\right)_{t \geq 0}$, whose generator $L$ stands in the right hand side of the equation, corresponds to a Markov process $(\xi(t))_{t \geq 0}$. Then this time-fractional Fokker-Planck-Kolmogorov equation is a governing equation for the stochastic process $\left(\xi\left(E_{t}^{\mu}\right)\right)_{t \geq 0}$ which is the time-change of $(\xi(t))_{t \geq 0}$ by means of the inverse subordinator $\left(E_{t}^{\mu}\right)_{t \geq 0}$. And the function

$$
f(t, x):=\mathbb{E}\left[f_{0}\left(\xi\left(E_{t}^{\mu}\right)\right) \mid \xi\left(E_{0}^{\mu}\right)=x\right]
$$

solves the Cauchy problem (22) (cf. Theorem 3.6 in 36, see also 3, 45]). Since the process $\left(\xi\left(E_{t}^{\mu}\right)\right)_{t \geq 0}$ is not Markov, its marginal density function (together with the initial distribution) does not determine all finite dimensional distributions of the process. And there exist many different processes with the same marginal density function. Hence there exist many other stochastic representations for the function $f(t, x)$ in formula (24) (see, e.g., Thm 3.3 in [1], Cor. 3.4 in [4] and results of 63). Furthermore, the considered time-fractional Fokker-Planck-Kolmogorov

\footnotetext{
${ }^{10}$ I.e., for all $\varphi \in X$, the mappings $t \mapsto\|F(t) \varphi\|_{X}$ from $[0, \infty)$ to $\mathbb{R}$ are Borel measurable. This is fulfilled if, e.g., the family $(F(t))_{t \geq 0}$ is strongly continuous. And if the family $(F(t))_{t \geq 0}$ of contractions on $X$ is such that all the mappings $t \mapsto\|F(t) \varphi\|_{X}, \varphi \in X$, are Borel measurable, then Bochner integrals in (23) are well-defined and finite.
} 
equations (with $\left.\mu=\delta_{\beta_{0}}, \beta_{0} \in(0,1)\right)$ are related to some time-non-fractional evolution equations of hihger order (see, e.g., 44, 64]). Therefore, the approximations $f_{n}$ constructed in Theorem 4.1 can be used simultaneousely to approximate path integrals appearing in different stochastic representations of the same function $f(t, x)$ and to approximate solutions of corresponding time-non-fractional evolution equations of hihger order.

Remark 4.2. Obviousely, approximations $f_{n}$ similar to those of Theorem 4.1 can be constructed also for subordinate semigroups discussed in Section 3.1 of [16. Namely, assume that a semigroup $\left(T_{t}^{f}\right)_{t \geq 0}$ is subordinate to a given semigroup $\left(T_{t}\right)_{t \geq 0}$ on a Banach space $\left(X,\|\cdot\|_{X}\right)$ with respect to a given convolution semigroup $\left(\eta_{t}\right)_{t \geq 0}$ associated to a Bernstein function $f$, i.e. $T_{t}^{f} \varphi=\int_{0}^{\infty} T_{s} \varphi \eta_{t}(d s)$ for all $\varphi \in X$; the convolution semigroup $\left(\eta_{t}\right)_{t \geq 0}$ is known explicitly; and a given family $(F(t))_{t \geq 0}$ (of contractions) is Chernoff equivalent to $\left(T_{t}\right)_{t \geq 0}$ and strongly Borel measurable. Then, similarly to the proof of Theorem 4.1. one shows that the functions $f_{n}(t)$,

$$
f_{n}(t):=\int_{0}^{\infty} F^{n}(s / n) f_{0} \eta_{t}(d s),
$$

approximate the function $T_{t}^{f} f_{0}$ in the norm $\|\cdot\|_{X}$ locally uniformly with respect to $t \geq 0$ for all $f_{0} \in \operatorname{Dom}(L)$. Note that such approximations $f_{n}$ are much simpler than Chernoff approximations based on the family $(\mathcal{F}(t))_{t \geq 0}$ constructed in Theorem 3.1 of [16]. However, the Chernoff approximations, based on the families $(\mathcal{F}(t))_{t \geq 0}$ which are presented in Theorem 3.1 of [16, can be used as a building block for further purposes. First, families $(\mathcal{F}(t))_{t \geq 0}$ can be used to obtain Chernoff approximations for semigroups, constructed by several iterative procedures of subordination, killing of an underlying process upon leaving a given domain, additive and multiplicative perturbations (of generators) of some original semigroups. Second, such Chernoff approximations, in turn, can be used to obtain approximations for solutions of the corresponding time-fractional evolution equations. Whereas the approximations $f_{n}$ in formula (25) can be used only to approximate $T_{t}^{f} f_{0}$ and not for further purposes.

4.2. Feynman formula solving the Cauchy problem for a class of timefractional diffusion equations. Let us return to the situation described in Corollary 3.1. So, let $\left(T_{t}\right)_{t \geq 0}$ be a Feller semigroun 11 whose generator $(L, \operatorname{Dom}(L))$ is given for all $\varphi \in C_{0}^{2}\left(\mathbb{R}^{d}\right)$ by formula (8). Let coefficients $A, B, C$ be bounded and continuous and (9) hold. Let $C_{c}^{2, \alpha}\left(\mathbb{R}^{d}\right)$ be a core for $(L, \operatorname{Dom}(L))$. Consider the family $(F(t))_{t \geq 0}$ of bounded linear operators on $X$ given by formula (17). Under the assumptions above, this family is strongly continuous and Chernoff equivalent to the semigroup $\left(T_{t}\right)_{t \geq 0}$. And all operators $F(t)$ are contractions since $C(x) \geq 0$ for all $x \in \mathbb{R}^{d}$. Let $f_{0} \in \operatorname{Dom}(L)$. Due to Proposition 4.1] the function

$$
f(t, x):=\int_{0}^{\infty} T_{\tau} f_{0}(x) p^{\mu}(t, \tau) d \tau
$$

solves the Cauchy problem for the distributed order time-fractional diffusion equation

$$
\mathcal{D}^{\mu} f(t, x)=-C(x) f(t, x)-B(x) \cdot \nabla f(t, x)+\operatorname{tr}(A(x) \operatorname{Hess} f(t, x))
$$

with the initial condition $f(0, x)=f_{0}(x)$. Due to Theorem 4.1, the following functions $f_{n}(t, x)$ approximate the solution $f(t, x)$ in supremum norm with respect

\footnotetext{
${ }^{11}$ Hence all $T_{t}$ are contractions.
} 
to $x \in \mathbb{R}^{d}$ uniformly with respect to $t \in\left(0, t^{*}\right]$ for all $t^{*}>0$ as $n \rightarrow \infty$ :

$$
\begin{aligned}
f_{n}\left(t, x_{0}\right):= & \int_{0}^{\infty} \int_{\mathbb{R}^{n d}} e^{-\frac{\tau}{n} \sum_{j=1}^{n}\left(C\left(x_{j-1}\right)+\frac{1}{4}\left|A^{-1 / 2}\left(x_{j-1}\right) B\left(x_{j-1}\right)\right|^{2}\right)} e^{\frac{1}{2} \sum_{j=1}^{n} A^{-1}\left(x_{j-1}\right) B\left(x_{j-1}\right) \cdot\left(x_{j-1}-x_{j}\right)} \\
& \times p_{A}\left(\tau / n, x_{0}, x_{1}\right) \cdots p_{A}\left(\tau / n, x_{n-1}, x_{n}\right) f_{0}\left(x_{n}\right) p^{\mu}(t, \tau) d x_{1} \ldots d x_{n} d \tau .
\end{aligned}
$$

Since for each $x_{0} \in \mathbb{R}^{d}$ the solution $f\left(t, x_{0}\right)$ is the limit of $f_{n}\left(t, x_{0}\right)$, i.e. the limit of $(n+1)$-fold iterated integrals as $n \rightarrow \infty$, the approximations $f_{n}\left(t, x_{0}\right)$ provide us just a Feynman formula for $f\left(t, x_{0}\right)$. Namely, the following statement holds.

Proposition 4.2. Under assumptions of this Subsection, the function $f\left(t, x_{0}\right)$, given by the Feynman formula (27) below, solves the Cauchy problem for the distributed order time-fractional diffusion equation (26) with the initial condition $f_{0}$.

$$
\begin{aligned}
& f\left(t, x_{0}\right) \\
& =\lim _{n \rightarrow \infty} \int_{0}^{\infty} \int_{\mathbb{R}^{n d}} e^{-\frac{\tau}{n} \sum_{j=1}^{n}\left(C\left(x_{j-1}\right)+\frac{1}{4}\left|A^{-1 / 2}\left(x_{j-1}\right) B\left(x_{j-1}\right)\right|^{2}\right)} e^{\frac{1}{2} \sum_{j=1}^{n} A^{-1}\left(x_{j-1}\right) B\left(x_{j-1}\right) \cdot\left(x_{j-1}-x_{j}\right)} \\
& \quad \times p_{A}\left(\tau / n, x_{0}, x_{1}\right) \cdots p_{A}\left(\tau / n, x_{n-1}, x_{n}\right) f_{0}\left(x_{n}\right) p^{\mu}(t, \tau) d x_{1} \ldots d x_{n} d \tau,
\end{aligned}
$$

where $p_{A}$ is given by (18). And the convergence is uniform with respect to $x_{0} \in \mathbb{R}^{d}$ and with respect to $t \in\left(0, t^{*}\right]$ for all $t^{*}>0$.

In particular, consider a $1 / 2$-stable inverse subordinator $\left(E_{t}^{1 / 2}\right)_{t \geq 0}$. Its marginal probability density is known explicitly (see Cor. 3.1 in 45 and the discussion after Lemma 3 in [28]

$$
p^{1 / 2}(t, \tau)=\frac{1}{\sqrt{\pi t}} e^{-\frac{\tau^{2}}{4 t}} .
$$

Therefore, in the case when $\mathcal{D}^{\mu}$ is the Caputo derivative of $1 / 2$-th order, the function $f\left(t, x_{0}\right)$, represented (uniformly with respect to $x_{0} \in \mathbb{R}^{d}$ and with respect to $t \epsilon$ $\left(0, t^{*}\right.$ for all $\left.t^{*}>0\right)$ by the following Feynman formula (28), solves the Cauchy problem for the time-fractional diffusion equation (26) with initial condition $f_{0}$ :

$$
\begin{aligned}
& f\left(t, x_{0}\right) \\
& =\lim _{n \rightarrow \infty} \int_{0}^{\infty} \int_{\mathbb{R}^{n d}} e^{-\frac{\tau}{n} \sum_{j=1}^{n}\left(C\left(x_{j-1}\right)+\frac{1}{4}\left|A^{-1 / 2}\left(x_{j-1}\right) B\left(x_{j-1}\right)\right|^{2}\right)} e^{\frac{1}{2} \sum_{j=1}^{n} A^{-1}\left(x_{j-1}\right) B\left(x_{j-1}\right) \cdot\left(x_{j-1}-x_{j}\right)} \\
& \quad \times p_{A}\left(\tau / n, x_{0}, x_{1}\right) \cdots p_{A}\left(\tau / n, x_{n-1}, x_{n}\right) f_{0}\left(x_{n}\right) \frac{1}{\sqrt{\pi t}} e^{-\frac{\tau^{2}}{4 t}} d x_{1} \ldots d x_{n} d \tau .
\end{aligned}
$$

Analogous results hold true for distributed order time-fractional Fokker-PlanckKolmogorov equations with non-local operators $L$ considered in Subsection 3.2 and in [16, 21].

4.3. Feynman formula solving the Cauchy-Dirichlet problem for a class of time-fractional diffusion equations. We keep on working in the situation of Subsection 4.2. Let additionally $\left(T_{t}\right)_{t \geq 0}$ be doubly Feller, $A, B, C$ be of the class $C_{b}^{2, \alpha}\left(\mathbb{R}^{d}\right)$ for some $\alpha \in(0,1)$. Let $G \subset \mathbb{R}^{d}$ be a bounded domain with the boundary $\partial G$ of the class $C^{4, \alpha}$ for some $\alpha \in(0,1)$. Then we have by Theorem 3.1 and Remark 3.2 for the corresponding semigroup $\left(T_{t}^{o}\right)_{t \geq 0}$ on $Y$

$$
T_{t}^{o} \varphi(x)=\lim _{n \rightarrow \infty}\left(\left(F_{o}(t / n)\right)^{n} \varphi\right)(x) \text { for all } \varphi \in C_{0}(G)
$$


uniformly in $x \in G$ and locally uniformly in $t \in[0, \infty)$. Here the family $\left(F_{o}(t)\right)_{t \geq 0}$ has been constructed from the family $(F(t))_{t \geq 0}$ given in (17) by the formula (11). Let $f_{0} \in \operatorname{Dom}\left(L_{o}\right)$. Due to Proposition 4.1 and Remark 2.1, the function

$$
f(t, x):=\int_{0}^{\infty} T_{\tau}^{o} f_{0}(x) p^{\mu}(t, \tau) d \tau
$$

solves the following Cauchy-Dirichlet problem for the distributed order time-fractional diffusion equation

$$
\begin{aligned}
& \mathcal{D}^{\mu} f(t, x)=-C(x) f(t, x)-B(x) \cdot \nabla f(t, x)+\operatorname{tr}(A(x) \operatorname{Hess} f(t, x)), \quad t>0, x \in G, \\
& f(0, x)=f_{0}(x), \quad x \in G, \\
& f(t, x)=0, \quad t>0, x \in \partial G .
\end{aligned}
$$

Due to Theorem 4.1 the following functions $f_{n}(t, x)$ approximate the solution $f(t, x)$ in supremum norm with respect to $x \in G$ uniformly with respect to $t \in\left(0, t^{*}\right]$ for all $t^{*}>0$ :

$$
\begin{aligned}
& f_{n}\left(t, x_{0}\right):=\int_{0}^{\infty} F_{o}^{n}(\tau / n) f_{0}\left(x_{0}\right) p^{\mu}(t, \tau) d \tau \\
& =\int_{0}^{\infty} \int_{\mathbb{R}^{n d}} e^{-\frac{\tau}{n} \sum_{j=1}^{n}\left(C\left(x_{j-1}\right)+\frac{1}{4}\left|A^{-1 / 2}\left(x_{j-1}\right) B\left(x_{j-1}\right)\right|^{2}\right)} e^{\frac{1}{2} \sum_{j=1}^{n} A^{-1}\left(x_{j-1}\right) B\left(x_{j-1}\right) \cdot\left(x_{j-1}-x_{j}\right)} \mathcal{E}\left(f_{0}\right)\left(x_{n}\right) \\
& \times\left(\prod_{j=1}^{n} \phi_{s(\tau / n)}\left(x_{j-1}\right)\right) p_{A}\left(\tau / n, x_{0}, x_{1}\right) \cdots p_{A}\left(\tau / n, x_{n-1}, x_{n}\right) p^{\mu}(t, \tau) d x_{1} \ldots d x_{n} d \tau .
\end{aligned}
$$

For each $\tau \in[0, \infty)$, each $x_{0} \in G$ and each $n \in \mathbb{N}$, let us define $\Theta_{n}^{f_{0}}\left(\tau, x_{0}\right)$ by

$$
\begin{aligned}
\Theta_{n}^{f_{0}}\left(\tau, x_{0}\right):=\int_{G^{n}} & e^{-\frac{\tau}{n} \sum_{j=1}^{n}\left(C\left(x_{j-1}\right)+\frac{1}{4}\left|A^{-1 / 2}\left(x_{j-1}\right) B\left(x_{j-1}\right)\right|^{2}\right)} e^{\frac{1}{2} \sum_{j=1}^{n} A^{-1}\left(x_{j-1}\right) B\left(x_{j-1}\right) \cdot\left(x_{j-1}-x_{j}\right)} \\
& \times f_{0}\left(x_{n}\right) p_{A}\left(\tau / n, x_{0}, x_{1}\right) \cdots p_{A}\left(\tau / n, x_{n-1}, x_{n}\right) d x_{1} \ldots d x_{n} .
\end{aligned}
$$

Then we have $\sup _{x_{0} \in G}\left|\Theta_{n}^{f_{0}}\left(\tau, x_{0}\right)\right| \leq\left\|f_{0}\right\|_{Y}$ for all $\tau \in[0, \infty)$ and $n \in \mathbb{N}$. Let us show that the functions

$$
g_{n}\left(t, x_{0}\right):=\int_{0}^{\infty} \Theta_{n}^{f_{0}}\left(\tau, x_{0}\right) p^{\mu}(t, \tau) d \tau
$$

approximate the function $f\left(t, x_{0}\right)$ in formula (29) solving the Cauchy-Dirichlet problem (30) as $n \rightarrow \infty$ locally uniformly with respect to $x_{0} \in G$ and uniformly with respect to $t \in\left(0, t^{*}\right]$ for all $t^{*}>0$. So, fix any $\varepsilon>0, T>0$ and a compact $K \subset G$. Let $x_{0} \in K$ and $t \in[0, T]$. Due to Lemma 4.1. there exists $R_{T, \varepsilon}>0$ such that $\int_{R_{T, \varepsilon}}^{\infty} p^{\mu}(t, \tau) d \tau<\varepsilon$ for all $t \in[0, T]$. Then, similarly to the proof of Theorem 4.1.

$$
\begin{aligned}
& \left|g_{n}\left(t, x_{0}\right)-f\left(t, x_{0}\right)\right| \leq\left|g_{n}\left(t, x_{0}\right)-f_{n}\left(t, x_{0}\right)\right|+\left|f_{n}\left(t, x_{0}\right)-f\left(t, x_{0}\right)\right| \\
& \leq \int_{0}^{R_{T, \varepsilon}}\left\|\Theta_{n}^{f_{0}}(\tau, \cdot)-F_{o}^{n}(\tau / n) f_{0}\right\|_{C(K)} p^{\mu}(t, \tau) d \tau+2 \varepsilon\left\|f_{0}\right\|_{Y}+\left\|f_{n}(t, \cdot)-f(t, \cdot)\right\|_{Y} \\
& \leq \sup _{\tau \in\left[0, R_{T, \varepsilon}\right]}\left\|\Theta_{n}^{f_{0}}(\tau, \cdot)-F_{o}^{n}(\tau / n) f_{0}\right\|_{C(K)}+2 \varepsilon\left\|f_{0}\right\|_{Y}+\left\|f_{n}(t, \cdot)-f(t, \cdot)\right\|_{Y} \\
& \rightarrow 2 \varepsilon\left\|f_{0}\right\|_{Y} \quad \text { as } n \rightarrow \infty,
\end{aligned}
$$

since the convergence of $\Theta_{n}^{f_{0}}\left(\tau, x_{0}\right)$ to $F_{o}^{n}(\tau / n) f_{0}\left(x_{0}\right)$ is locally uniform with respect to $x_{0} \in G$ and uniform with respect to $t \in\left(0, t^{*}\right]$ for all $t^{*}>0$ (cf. Corollary 3.1) and 
due to Theorem 4.1. Therefore, since $\varepsilon>0$ is arbitrary, the following statement is proved.

Proposition 4.3. Let $\left(T_{t}\right)_{t \geq 0}$ be a doubly Feller semigroup on $X=C_{0}\left(\mathbb{R}^{d}\right)$ whose generator $(L, \operatorname{Dom}(L))$ is given for all $\varphi \in C_{0}^{2}\left(\mathbb{R}^{d}\right)$ by the formula (8). Let the coefficients $A, B, C$ in (8) be of the class $C_{b}^{2, \alpha}\left(\mathbb{R}^{d}\right)$ for some $\alpha \in(0,1)$. Let there exist $a_{0}, A_{0} \in \mathbb{R}$ such that (9) holds. Assume that the coefficients $A, B, C$ are such that the set $C_{c}^{2, \alpha}\left(\mathbb{R}^{d}\right)$ is a core for the generator $L$ in $X$. Let $G \subset \mathbb{R}^{d}$ be a bounded domain with the boundary $\partial G$ of the class $C^{4, \alpha}$. Let $\left(T_{t}^{o}\right)_{t \geq 0}$ be the corresponding Feller semigroup on $Y=C_{0}(G)$ with the generator $\left(L_{o}, \operatorname{Dom}\left(L_{o}\right)\right)$. Let $f_{0} \in \operatorname{Dom}\left(L_{o}\right)$. Let $\mu$ be a finite Borel measure with $\operatorname{supp} \mu \in(0,1)$. Then the function $f\left(t, x_{0}\right)$, which is given for all $t>0$ and all $x_{0} \in G$ by the Feynman formula (31) below, solves the Cauchy-Dirichlet problem (30). And the convergence in the Feynman formula (31) is locally uniform with respect to $x_{0} \in G$ and uniform with respect to $t \in\left(0, t^{*}\right]$ for all $t^{*}>0$ :

$$
\begin{aligned}
& f\left(t, x_{0}\right) \\
& =\lim _{n \rightarrow \infty} \int_{0}^{\infty} \int_{G^{n}} e^{-\frac{\tau}{n} \sum_{j=1}^{n}\left(C\left(x_{j-1}\right)+\frac{1}{4}\left|A^{-1 / 2}\left(x_{j-1}\right) B\left(x_{j-1}\right)\right|^{2}\right)} e^{\frac{1}{2} \sum_{j=1}^{n} A^{-1}\left(x_{j-1}\right) B\left(x_{j-1}\right) \cdot\left(x_{j-1}-x_{j}\right)} \\
& \quad \times p_{A}\left(\tau / n, x_{0}, x_{1}\right) \cdots p_{A}\left(\tau / n, x_{n-1}, x_{n}\right) f_{0}\left(x_{n}\right) p^{\mu}(t, \tau) d x_{1} \ldots d x_{n} d \tau,
\end{aligned}
$$

where $p_{A}$ is given by (18).

Analogous results hold true for distributed order time-fractional Fokker-PlanckKolmogorov equations with non-local operators $L$ considered in Subsection 3.2 Other representations for solutions of some distributed order time-fractional FokkerPlanck-Kolmogorov equations in bounded domains can be found, e.g., in [44, 24].

\section{ACKNOWLEDGEMENTS}

I would like to thank Christian Bender for the encouragement and support of my researches; Alessandra Lunardi for her remarks (they are presented in Remark 3.1) on properties of the Laplace operator with Dirichlet boundary conditions; Krzysztof Bogdan for communicating me the reference [2] and Panki Kim for remarks on killed Feller processes.

\section{REFERENCES}

[1] B. Baeumer, M. Kovács, M. M. Meerschaert, R. L. Schilling, and P. Straka. Reflected spectrally negative stable processes and their governing equations. Trans. Amer. Math. Soc., 368(1):227-248, 2016.

[2] B. Baeumer, T. Luks, and M. M. Meerschaert. Space-time fractional Dirichlet problems. Preprint, 2016.

[3] B. Baeumer and M. M. Meerschaert. Stochastic solutions for fractional Cauchy problems. Fract. Calc. Appl. Anal., 4(4):481-500, 2001.

[4] B. Baeumer, M. M. Meerschaert, and E. Nane. Brownian subordinators and fractional Cauchy problems. Trans. Amer. Math. Soc., 361(7):3915-3930, 2009.

[5] B. Baur, F. Conrad, and M. Grothaus. Smooth contractive embeddings and application to Feynman formula for parabolic equations on smooth bounded domains. Comm. Statist. Theory Methods, 40(19-20):3452-3464, 2011.

[6] K. Bogdan, K. Burdzy, and Z.-Q. Chen. Censored stable processes. Probab. Theory Related Fields, 127(1):89-152, 2003.

[7] B. Böttcher, Y. A. Butko, R. L. Schilling, and O. G. Smolyanov. Feynman formulas and path integrals for some evolution semigroups related to $\tau$-quantization. Russ. J. Math. Phys., 18(4):387-399, 2011. 
[8] B. Böttcher, R. Schilling, and J. Wang. Lévy matters. III, volume 2099 of Lecture Notes in Mathematics. Springer, Cham, 2013. Lévy-type processes: construction, approximation and sample path properties, With a short biography of Paul Lévy by Jean Jacod, Lévy Matters.

[9] B. Böttcher and R. L. Schilling. Approximation of Feller processes by Markov chains with Lévy increments. Stoch. Dyn., 9(1):71-80, 2009.

[10] Y. A. Butko. Representations of the solution of the Cauchy-Dirichlet problem for the heat equation in a domain of a compact Riemannian manifold by functional integrals. Russ. J. Math. Phys., 11(2):121-129, 2004.

[11] Y. A. Butko. Functional integrals corresponding to a solution of the Cauchy-Dirichlet problem for the heat equation in a domain of a Riemannian manifold. Fundam. Prikl. Mat., 12(6):3-15, 2006.

[12] Y. A. Butko. Functional integrals over Smolyanov surface measures for evolutionary equations on a Riemannian manifold. In Quantum probability and infinite dimensional analysis, volume 20 of $Q P-P Q$ : Quantum probability and infinite dimensional analysis, pages 145-155. World Sci. Publ., Hackensack, NJ, 2007.

[13] Y. A. Butko. Feynman formulas and functional integrals for diffusion with drift in a domain on a manifold. Mat. Zametki, 83(3):333-349, 2008.

[14] Y. A. Butko. Feynman formulae for evolution semigroups. Scientific periodical of the Bauman MSTU 'Science and Education", 3:95-132, 2014.

[15] Y. A. Butko. Description of quantum and classical dynamics via Feynman formulae. In Mathematical results in quantum mechanics, pages 227-233. World Sci. Publ., Hackensack, NJ, 2015.

[16] Y. A. Butko. Chernoff approximations for subordinate semigroups. Stochastics and Dynamics, pages 1850021, 19, 2017.

[17] Y. A. Butko, M. Grothaus, and O. G. Smolyanov. Feynman's formula for a second-order parabolic equation in a domain. Dokl. Akad. Nauk, 421(6):727-732, 2008.

[18] Y. A. Butko, M. Grothaus, and O. G. Smolyanov. Lagrangian Feynman formulas for secondorder parabolic equations in bounded and unbounded domains. Infin. Dimens. Anal. Quantum Probab. Relat. Top., 13(3):377-392, 2010.

[19] Y. A. Butko, M. Grothaus, and O. G. Smolyanov. Feynman formulae and phase space Feynman path integrals for tau-quantization of some Lévy-Khintchine type Hamilton functions. J. Math. Phys., 57(2):023508, 22, 2016.

[20] Y. A. Butko, R. L. Schilling, and O. G. Smolyanov. Hamiltonian Feynman-Kac and Feynman formulae for dynamics of particles with position-dependent mass. Internat. J. Theoret. Phys., 50(7):2009-2018, 2011.

[21] Y. A. Butko, R. L. Schilling, and O. G. Smolyanov. Lagrangian and Hamiltonian Feynman formulae for some Feller semigroups and their perturbations. Infin. Dimens. Anal. Quantum Probab. Relat. Top., 15(3):26, 2012.

[22] Y. A. Butko, O. G. Smolyanov, and R. L. Schilling. Feynman formulas for Feller semigroups. Dokl. Akad. Nauk, 434(1):7-11, 2010.

[23] L. Chen, E. R. Jakobsen, and A. Naess. On numerical density approximations of solutions of sdes with unbounded coefficients. Preprint, 2016.

[24] Z.-Q. Chen, M. M. Meerschaert, and E. Nane. Space-time fractional diffusion on bounded domains. J. Math. Anal. Appl., 393(2):479-488, 2012.

[25] Z.-Q. Chen and R. Song. Intrinsic ultracontractivity and conditional gauge for symmetric stable processes. J. Funct. Anal., 150(1):204-239, 1997.

[26] P. R. Chernoff. Note on product formulas for operator semigroups. J. Functional Analysis, 2:238-242, 1968.

[27] P. R. Chernoff. Product formulas, nonlinear semigroups, and addition of unbounded operators. American Mathematical Society, Providence, R. I., 1974. Memoirs of the American Mathematical Society, No. 140.

[28] M. D'Ovidio. Explicit solutions to fractional diffusion equations via generalized gamma convolution. Electron. Commun. Probab., 15:457-474, 2010.

[29] M. Felsinger, M. Kassmann, and P. Voigt. The Dirichlet problem for nonlocal operators. Math. Z., 279(3-4):779-809, 2015.

[30] M. Gadèl'ya and O. G. Smolyanov. Feynman formulas for particles with position-dependent mass. Dokl. Akad. Nauk, 418(6):727-730, 2008

[31] R. Garra, E. Orsingher, and F. Polito. Fractional diffusions with time-varying coefficients. J. Math. Phys., 56(9):093301, 17, 2015.

[32] J. E. Gillis and G. H. Weiss. Expected number of distinct sites visited by a random walk with an infinite variance. J. Mathematical Phys., 11:1307-1312, 1970.

[33] R. Gorenflo and F. Mainardi. Random walk models for space-fractional diffusion processes. Fract. Calc. Appl. Anal., 1(2):167-191, 1998. 
[34] J. Gough, O. O. Obrezkov, and O. G. Smolyanov. Randomized Hamiltonian Feynman integrals and stochastic Schrödinger-Itô equations. Izv. Ross. Akad. Nauk Ser. Mat., 69(6):3-20, 2005.

[35] M. Hahn, K. Kobayashi, and S. Umarov. Fokker-Planck-Kolmogorov equations associated with time-changed fractional Brownian motion. Proc. Amer. Math. Soc., 139(2):691-705, 2011.

[36] M. Hahn, K. Kobayashi, and S. Umarov. SDEs driven by a time-changed Lévy process and their associated time-fractional order pseudo-differential equations. Journal of Theoretical Probability, 25(1):262-279, 2012.

[37] M. Hahn and S. Umarov. Fractional Fokker-Planck-Kolmogorov type equations and their associated stochastic differential equations. Fract. Calc. Appl. Anal., 14(1):56-79, 2011.

[38] W. Hoh and N. Jacob. On the Dirichlet problem for pseudodifferential operators generating Feller semigroups. J. Funct. Anal., 137(1):19-48, 1996.

[39] N. Jacob. Pseudo differential operators and Markov processes. Vol. I. Imperial College Press, London, 2001. Fourier analysis and semigroups.

[40] A. N. Kochubei. Distributed order calculus and equations of ultraslow diffusion. J. Math. Anal. Appl., 340(1):252-281, 2008.

[41] J. Kupsch and O. G. Smolyanov. Generalized Wiener-Segal-Fock representations and Feynman formulas. Dokl. Akad. Nauk, 425(1):15-19, 2009.

[42] A. Lunardi. Analytic semigroups and optimal regularity in parabolic problems. Modern Birkhäuser Classics. Birkhäuser/Springer Basel AG, Basel, 1995.

[43] F. Mainardi, A. Mura, G. Pagnini, and R. Gorenflo. Time-fractional diffusion of distributed order. J. Vib. Control, 14(9-10):1267-1290, 2008.

[44] M. M. Meerschaert, E. Nane, and P. Vellaisamy. Distributed-order fractional diffusions on bounded domains. J. Math. Anal. Appl., 379(1):216-228, 2011.

[45] M. M. Meerschaert and H.-P. Scheffler. Limit theorems for continuous-time random walks with infinite mean waiting times. J. Appl. Probab., 41(3):623-638, 2004.

[46] M. M. Meerschaert and H.-P. Scheffler. Stochastic model for ultraslow diffusion. Stochastic Process. Appl., 116(9):1215-1235, 2006.

[47] M. M. Meerschaert and H.-P. Scheffler. Triangular array limits for continuous time random walks. Stochastic Process. Appl., 118(9):1606-1633, 2008.

[48] M. M. Meerschaert and A. Sikorskii. Stochastic models for fractional calculus, volume 43 of De Gruyter Studies in Mathematics. Walter de Gruyter \& Co., Berlin, 2012.

[49] M. M. Meerschaert and P. Straka. Inverse stable subordinators. Math. Model. Nat. Phenom., $8(2): 1-16,2013$.

[50] R. Metzler and J. Klafter. The random walk's guide to anomalous diffusion: a fractional dynamics approach. Phys. Rep., 339(1):77, 2000.

[51] R. Metzler and J. Klafter. The restaurant at the end of the random walk: recent developments in the description of anomalous transport by fractional dynamics. J. Phys. A, 37(31):R161R208, 2004.

[52] J. B. Mijena and E. Nane. Strong analytic solutions of fractional Cauchy problems. Proc. Amer. Math. Soc., 142(5):1717-1731, 2014.

[53] K. S. Miller and B. Ross. An introduction to the fractional calculus and fractional differential equations. A Wiley-Interscience Publication. John Wiley \& Sons, Inc., New York, 1993.

[54] E. W. Montroll and M. F. Shlesinger. On the wonderful world of random walks. In Nonequilibrium phenomena, II, Stud. Statist. Mech., XI, pages 1-121. North-Holland, Amsterdam, 1984.

[55] A. Mura, M. S. Taqqu, and F. Mainardi. Non-Markovian diffusion equations and processes: analysis and simulations. Phys. A, 387(21):5033-5064, 2008.

[56] O. O. Obrezkov. The proof of the Feynman-Kac formula for heat equation on a compact Riemannian manifold. Infin. Dimens. Anal. Quantum Probab. Relat. Top., 6(2):311-320, 2003.

[57] O. O. Obrezkov. Representation of a solution of a stochastic Schrödinger equation in the form of a Feynman integral. Fundam. Prikl. Mat., 12(5):135-152, 2006.

[58] O. O. Obrezkov and O. G. Smolyanov. Representations of the solutions of Lindblad equations with the help of randomized Feynman formulas. Dokl. Akad. Nauk, 466(5):518-521, 2016.

[59] O. O. Obrezkov, O. G. Smolyanov, and A. Trumen. A generalized Chernoff theorem and a randomized Feynman formula. Dokl. Akad. Nauk, 400(5):596-601, 2005.

[60] Y. N. Orlov, V. Z. Sakbaev, and O. G. Smolyanov. Feynman formulas as a method of averaging random Hamiltonians. In Proceedings of the Steklov Institute of Mathematics, volume 285, pages 222-232. 2014. 
[61] Y. N. Orlov, V. Z. Sakbaev, and O. G. Smolyanov. Unbounded random operators and Feynman formulae. Rossiǔskaya Akademiya Nauk. Izvestiya. Seriya Matematicheskaya, 80(6):141$172,2016$.

[62] E. Orsingher and L. Beghin. Time-fractional telegraph equations and telegraph processes with Brownian time. Probab. Theory Related Fields, 128(1):141-160, 2004.

[63] E. Orsingher and L. Beghin. Fractional diffusion equations and processes with randomly varying time. Ann. Probab., 37(1):206-249, 2009.

[64] E. Orsingher and M. D'Ovidio. Probabilistic representation of fundamental solutions to $\frac{\partial u}{\partial t}=$ $\kappa_{m} \frac{\partial^{m} u}{\partial x^{m}}$. Electron. Commun. Probab., 17:no. 1885, 12, 2012.

[65] A. Pazy. Semigroups of linear operators and applications to partial differential equations, volume 44 of Applied Mathematical Sciences. Springer-Verlag, New York, 1983.

[66] A. S. Plyashechnik. Feynman formula for Schrödinger-type equations with time- and spacedependent coefficients. Russ. J. Math. Phys., 19(3):340-359, 2012.

[67] A. S. Plyashechnik. Feynman formulas for second-order parabolic equations with variable coefficients. Russ. J. Math. Phys., 20(3):377-379, 2013.

[68] J. Prüss. Evolutionary integral equations and applications. Modern Birkhäuser Classics. Birkhäuser/Springer Basel AG, Basel, 1993. [2012] reprint of the 1993 edition.

[69] T. S. Rat' yu and O. G. Smolyanov. Dynamics of particles with anisotropic mass that depends on time and position. Dokl. Akad. Nauk, 465(4):407-410, 2015.

[70] I. D. Remizov. Solution of a Cauchy problem for a diffusion equation in a Hilbert space by a Feynman formula. Russ. J. Math. Phys., 19(3):360-372, 2012.

[71] I. D. Remizov. Quasi-Feynman formulas a method of obtaining the evolution operator for the Schrödinger equation. Journal of Functional Analysis, 270(12):4540-4557, 2016.

[72] J. D. Rossi and E. Topp. Large solutions for a class of semilinear integro-differential equations with censored jumps. J. Differential Equations, 260(9):6872-6899, 2016.

[73] A. I. Saichev and G. M. Zaslavsky. Fractional kinetic equations: solutions and applications. Chaos, 7(4):753-764, 1997.

[74] V. Z. Sakbaev and O. G. Smolyanov. The dynamics of a quantum particle with discontinuous dependence of the mass on position. Dokl. Akad. Nauk, 433(3):314-317, 2010.

[75] S. G. Samko, A. A. Kilbas, and O. I. Marichev. Fractional integrals and derivatives. Gordon and Breach Science Publishers, Yverdon, 1993. Theory and applications, Edited and with a foreword by S. M. Nikol'skiı̌, Translated from the 1987 Russian original, Revised by the authors.

[76] O. G. Smolyanov and N. N. Shamarov. Feynman and Feynman-Kac formulas for evolution equations with the Vladimirov operator. Dokl. Akad. Nauk, 420(1):27-32, 2008.

[77] O. G. Smolyanov and N. N. Shamarov. Feynman formulas and path integrals for evolution equations with the Vladimirov operator. Tr. Mat. Inst. Steklova, 265(Izbrannye Voprosy Matematicheskoy Fiziki i p-adicheskogo Analiza):229-240, 2009.

[78] O. G. Smolyanov and N. N. Shamarov. Hamiltonian Feynman integrals for equations with the Vladimirov operator. Dokl. Akad. Nauk, 431(2):170-174, 2010.

[79] O. G. Smolyanov and N. N. Shamarov. Hamiltonian Feynman formulas for equations containing the Vladimirov operator with variable coefficients. Dokl. Akad. Nauk, 440(5):597-602, 2011.

[80] O. G. Smolyanov, N. N. Shamarov, and M. Kpekpassi. Feynman-Kac and Feynman formulas for infinite-dimensional equations with the Vladimirov operator. Dokl. Akad. Nauk, 438(5):609-614, 2011.

[81] O. G. Smolyanov and E. T. Shavgulidze. Feynman formulas for solutions of infinitedimensional Schrödinger equations with polynomial potentials. Dokl. Akad. Nauk, 390(3):321-324, 2003.

[82] O. G. Smolyanov and D. S. Tolstyga. Feynman formulas for stochastic and quantum dynamics of particles in multidimensional domains. Dokl. Akad. Nauk, 452(3):256-260, 2013.

[83] O. G. Smolyanov and A. Truman. Feynman formulas for solutions of Schrödinger equations on compact Riemannian manifolds. Mat. Zametki, 68(5):789-793, 2000.

[84] O. G. Smolyanov and A. Truman. Hamiltonian Feynman formulas for the Schrödinger equation in bounded domains. Dokl. Akad. Nauk, 399(3):310-314, 2004.

[85] O. G. Smolyanov, H. von Weizsäcker, and O. Wittich. Feynman formulas for the Cauchy problem in domains with a boundary. Dokl. Akad. Nauk, 395(5):596-600, 2004.

[86] O. G. Smolyanov, H. von Weizsäcker, and O. Wittich. Surface measures and initial-boundary value problems generated by diffusions with drift. Dokl. Akad. Nauk, 415(6):737-741, 2007.

[87] S. Umarov and R. Gorenflo. Cauchy and nonlocal multi-point problems for distributed order pseudo-differential equations. I. Z. Anal. Anwendungen, 24(3):449-466, 2005. 
APPROXIMATIONS FOR KILLED PROCESSES AND TIME-FRACTIONAL EQUATIONS 25

[88] S. Umarov and R. Gorenflo. On multi-dimensional random walk models approximating symmetric space-fractional diffusion processes. Fract. Calc. Appl. Anal., 8(1):73-88, 2005.

[89] H. von Weizsäcker and O. G. Smolyanov. Feynman formulas generated by selfadjoint extensions of the Laplace operator. Dokl. Akad. Nauk, 426(2):162-165, 2009.

[90] H. von Weizsäcker, O. G. Smolyanov, and D. S. Tolstyga. Feynman description of the onedimensional dynamics of particles with piecewise-continuous dependence of mass on the coordinates. Dokl. Akad. Nauk, 441(3):295-298, 2011.

[91] G. M. Zaslavsky. Chaos, fractional kinetics, and anomalous transport. Phys. Rep., 371(6):461$580,2002$. 